\title{
CFAR Ship Detection in Polarimetric Synthetic Aperture Radar Images Based on Whitening Filter
}

\author{
Tao Liu, Jiafeng Zhang, Gui Gao, Member, IEEE, Jian Yang, Member, IEEE, and Armando Marino, Member, IEEE
}

\begin{abstract}
Polarimetric Whitening Filter (PWF) can be used to filter Polarimetric Synthetic Aperture (PolSAR) images to improve the contrast between ships and sea clutter background. For this reason, the output of the filter can be used to detect ships. This work deals with the setting of the threshold over PolSAR images filtered by the PWF.

Two parameter-constant false alarm rate (2P-CFAR) is a common detection method used on whitened polarimetric images. It assumes that the probability density function (PDF) of the filtered image intensity is characterized by a lognormal distribution. However, this assumption does not always hold. In this paper, we propose a systemic analytical framework for CFAR algorithms based on PWF or multi-look PWF (MPWF). The framework covers the entire log-cumulants space in terms of the textural distributions in the product model, including the constant, Gamma, Inverse Gamma, Fisher, Beta, inverse Beta and Generalized Gamma (GГD) distributions. We derive the analytical forms of the PDF for each of the textural distributions and the probability of false alarm (PFA). Finally, the threshold is derived by fixing the false alarm rate (FAR). Experimental results using both the simulated and real data demonstrate that the derived expressions and CFAR algorithms are valid and robust.
\end{abstract}

Index Terms-Constant False Alarm Rate, Polarimetric Whitening Filter, Radar Polarimetry, Synthetic Aperture Radar, Ship Detection

\section{INTRODUCTION}

$\mathrm{P}$ OLARIMETRIC Synthetic Aperture Radar (PolSAR) is a multi-dimensional remote sensing system which has been largely used for marine surveillance, including ship detection [1]. In SAR images, the main feature of ships is a relatively large backscattering signal, which is usually brighter compared to the sea background. This led to the idea of detecting vessels using a statistical test on the intensity (i.e. brightness) of the sea

This work was supported in part by the National Natural Science Foundation of China under Project 61771483 and 61490693.

T. Liu and J. F. Zhang are with the school of electronic engineering, Naval University of Engineering, Wuhan, 430033, China (e-mail:

liutao1018@hotmail.com).

G. Gao is with the College of Traffic Engineering, Hunan University of Technology, Zhuzhou, 412007, China, and with the Faculty of Geoscience and Environmental Engineering, Southwest Jiaotong University, Chengdu, 611756, China (e-mail: dellar@126.com).

$\mathrm{J}$. Yang is with the department of electronic engineering, Tsinghua University, Beijing, 100084, China.

A. Marino is with the Faculty of Natural Sciences, University of Stirling, UK (armando.marino@stir.ac.uk). clutter [1]. When the distributions of clutter and targets are both known, an optimal detector can be given by the likelihood ratio test (LRT). Based on this idea, Novak et al. proposed the optimal polarimetric detector (OPD) [2]-[3]. Unfortunately, the statistical distribution of ship backscattering is difficult to obtain, since this depends highly on the physical characteristics of the vessel. For this reason, tests are generally based only on the probability of false alarms (PFA) of sea clutter for simplification in ship detection. A common way selects the threshold that locally keeps PFA constant [1].

When polarimetric data are available the polarimetric information can be used to improve the detection performance. Recently, a Polarimetric Notch Filter (PNF) was presented that tries to separate ships and vessels based on their polarimetric behavior [4]-[5]. It does not assume a prior information about the ships and it was showed to provide good performance by minimizing the sea clutter power [1][6]-[7].

Polarimetric decompositions and other physical based models [8] have also been used for ship detection. Ringrose used the Cameron decomposition method to detect ships from SIR-C data [9]. Chen et al. proposed a polarization cross entropy for detecting ships [10]. Ship wakes provide useful information about ship heading and velocity, which can be used in ship detection [11]-[14]; however ship wake detection is influenced by various factors, such as sea state, radar frequency, angle of view and angle of incidence.

Another filter working in the absence of ship prior information to improve the ship detection performance is the Polarimetric Whitening Filter (PWF). The PWF uses the polarimetric information to minimize the statistical variation due to speckle. In early tests, its performance was the closest to that of the OPD, especially in the high target-clutter-ratio (TCR) case [3][15]. The PWF was further developed to Multi-look Polarimetric Whitening Filter (MPWF) by Lopes and Liu et al. [16]-[17]. The PWF can be seen as a special case of the MPWF. The MPWF was in the following extended MPWF (EMPWF) to filter the images while preserving the polarimetric information [18]. Anfinsen et al. modified the PWF and presented the fixed-point PWF (FPPWF) [19], which originates from the fixed-point estimator of the covariance matrix in the multivariate product model. Recently, Gao et al. proposed an Extended Polarimetric Whitening Filter (EPWF) for ship detection in PolSAR images in which clutter is nonhomogeneous and exhibits a channel-dependency [20].

In this work we focus on the MPWF since this has been shown to provide good performances which are close to the 
OPD [3][15]. Specifically, we are developing an algorithm able to set the threshold on filtered images using a CFAR. CFAR detectors try to adaptively determine the threshold based on accurate modeling and estimation of the statistical distribution of local background clutter. The distribution parameters are often estimated using sliding windows techniques [1][6]-[7]. Additionally, truncated methods can be applied to solve the multi targets problems inside the training window [21].

Different background clutter can have different statistical distributions. Therefore, different statistical models should be used for characterizing different marine regions in PolSAR images. Many PDFs have been proposed to model the statistical characteristics of the sea clutter [7][21]. In case of PWF images, a common assumption is that the probability density function (PDF) is a log-normal distribution [15]. Under this assumption, PFA were derived when the texture is constant (e.g. polarimetric covariance matrices can be modelled as Wishart distributed [17]) or obeys a gamma distribution (the corresponding covariance matrix is K-Wishart distributed) [22]. Since the expressions in Wishart model or K-Wishart model are complicated, it is relatively difficult to find analytically the threshold for the CFAR detector [17][22]. Additionally, in real scenarios, the intensity distribution may not obey a log-normal distribution.

Multivariate product models are proposed to model more complex sea clutter [3]. Besides constant or gamma distributions, the texture in product models may also obey inverse Gamma, Fisher, Beta, and inverse Beta. All these distributions can cover the entire log-cumulants space in terms of textural distributions [23]. In order to encompass all this models we can use the GГD (generalized gamma distribution) since it includes many kinds of distributions [24]. Fig1 shows the coverage of the presented distributions on the $k 2-k 3$ log-cumulants plane.

Although the MPWF has been introduced many years ago, work on deriving the analytical forms of the PFA and the corresponding threshold in different statistical models (the texture obeys inverse gamma, Fisher, Beta, inverse Beta or GГD distribution) is still limited and incomplete.

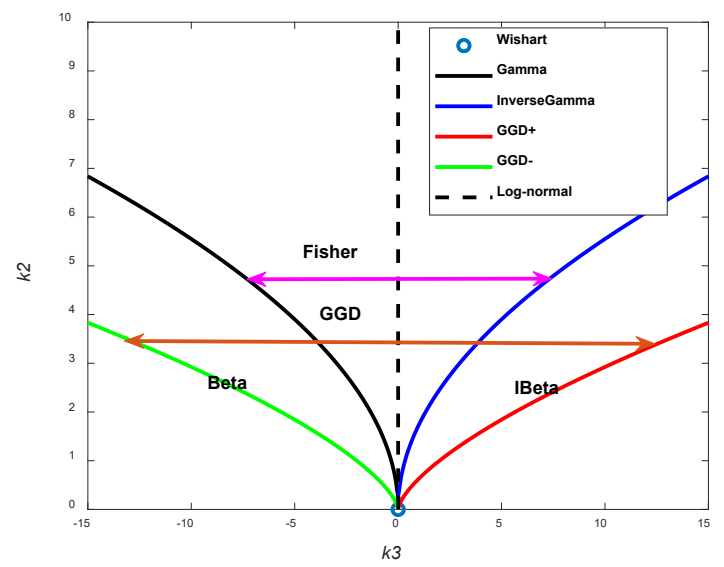

Fig. 1. Distributions displayed in the $(k 3, k 2)$ log-cumulants diagram: $k 3$ is the third order log-cumulants, and $k 2$ is the second order log-cumulants.

In addition, the existing expressions of PFA in Wishart or K-Wishart case [3][22] can only be applied in a CFAR when the Equivalent Number of Looks (ENL) is an integer. The ENL is a parameter that in some conditions estimates how many independent realizations have been averaged together to produce the image. In the practice, it is the parameter that produces the best match between empirical moments of the correlated data and theoretical moments of the data model, which assumes independence [25]. Unfortunately, the ENL is generally not an integer number [25]. For this reason, in this work we develop a new threshold estimation which can be applied to fractional number of looks.

To summarize, the main novelties of this work are: a) to exploit the constant, Gamma, Inverse Gamma, Fisher, Beta, inverse Beta and GГD distributed texture to derive the analytical expressions of the PDF and PFA of the MPWF; $b$ ) to use the previous expressions to set a novel CFAR test on the MPWF.

The paper is organized as follows: The basic concepts of data description, product model and MPWF in polarimetric SAR are briefly introduced in Section II. The closed forms of the PFAs are derived for CFAR processing in section III where the texture obeys a constant, gamma, inverse Gamma, Fisher, Beta, inverse Beta or GГD distribution. To obtain more accurate estimations of the statistical parameters, two novel parameter estimators are proposed based on the MPWF and log-cumulants for product models in Section IV. All the CFAR algorithms are verified via both the simulated data and measured data in Section V. Finally, the conclusions are given in Section VI.

\section{Statistical Model of Pol-SAR data}

\section{A. Description of Pol-SAR Data}

Under far-field assumptions, a polarimetric scattering matrix can represent the scattering characteristics of targets. When a Horizontal-Vertical linear basis is established, the polarimetric scattering matrix $\mathbf{S}$ can be represented [1][3]

$$
\mathbf{S}=\left[\begin{array}{ll}
S_{H H} & S_{H V} \\
S_{V H} & S_{V V}
\end{array}\right]
$$

where $S_{x y}$ represents the complex scattering coefficient with $x$ standing for the transmitting polarization, and $y$ the receiving polarization (H-horizontal linear, $\mathrm{V}$-vertical linear). When the system is monostatic and the reciprocity condition is satisfied, $S_{H V}=S_{V H}$. Then the scattering vector $\mathbf{k}$ can be defined as

$$
\mathbf{k}=\left[\begin{array}{c}
S_{H H} \\
\sqrt{2} S_{H V} \\
S_{V V}
\end{array}\right]
$$

To reduce the speckle in the SAR imagery, averages and multi-look processing can be applied. We can therefore obtain the covariance matrix $\mathbf{C}$ as [3][17]

$$
\mathbf{C}=\frac{1}{L} \sum_{i=1}^{L} \mathbf{k}_{i} \mathbf{k}_{i}^{\dagger}
$$

where $L$ is the number of averaged pixels (or looks). The superscript $\dagger$ denotes conjugate transpose. The multilook covariance matrix $\mathbf{C}$ is a random variable and therefore it can be modeled using a PDF. 


\section{B. Multivariate Product Model}

The backscattering of a SAR image presents statistical variation. Part of this variation is due to the interference between scatterers within a resolution cell which is also referred to as speckle. Part of the variation is due to the fluctuation of the underlying radar cross section (RCS) or speckle, referred to as texture [7]. The variation induced by texture is slower than the one induced by speckle in the sense that it produces a modulation that can only be observed on a large amount of pixels. The PDF of a covariance matrix which does not present texture (but only speckle) can be characterized using the Wishart distribution.

If texture is present, the model becomes more complicated and can be formulated by the well-known multiplicative model for two independent stochastic variables [7]. The polarimetric scattering vector $\mathbf{k}$ in the product model can be presented as [3]

$$
\mathbf{k}=\sqrt{\tau} \mathbf{y}
$$

where $\tau$ is the scalar texture variable, and $\mathbf{y}$ is the speckle vector, which follows a zero-mean multivariate complex Gaussian distribution. Please note that in this model each polarization channel is assumed to have the same texture.

The multilook covariance matrix $\mathbf{C}$ is [3]

$$
\mathbf{C}=\frac{1}{L} \sum_{i=1}^{L} \mathbf{k}_{i} \mathbf{k}_{i}^{\dagger}=\frac{1}{L} \sum_{i=1}^{L} \tau_{i} \mathbf{y}_{i} \mathbf{y}_{i}^{\dagger}
$$

Since the variation of the texture variable $\tau$ is slower than the speckle $\mathbf{y}$ [26], the texture variable $\tau$ is often assumed to be constant in the multi-look process, and thus $\tau_{i}$ is independent of $i$. This means that the averaging window used to obtain $\mathbf{C}$ is not large enough to observe variations of $\tau_{i}$. Then, the above equation can be simplified as [3][27]

$$
\mathbf{C}=\tau \mathbf{Y}
$$

where $\mathbf{Y}$ is a random matrix only affected by speckle. Therefore $\mathbf{Y}$ obeys the Wishart distribution [2].

Since texture and speckle are independent, we can be obtained [27]

$$
\boldsymbol{\Sigma}=\mathrm{E}\{\mathbf{C}\}=\mathrm{E}\{\tau\} \mathrm{E}\{\mathbf{Y}\}=\mathrm{E}\{\tau\} \Gamma
$$

where $\mathrm{E}(\bullet)$ represents the expectation operator $\Gamma=E\left\{\mathbf{y y} \mathbf{y}^{\dagger}\right\}$ is the covariance matrix of the speckle in the Gaussian case, $\boldsymbol{\Sigma}$ is the statistical mean of the multi look covariance matrix $\mathbf{C}$.

\section{The Output of MPWF}

It is well known that synthetic aperture radar (SAR) produces images with speckle [3][17]. The presence of the speckle complicates image interpretation, degrades the image segmentation performance, and reduces the detectability of targets in the images. The availability of polarimetric SAR data has made it possible to reduce the image speckle by processing the different channels together[17]. Novak and Burl [3] proposed the polarimetric whitening filter (PWF) to reduce the speckle by using fully polarimetric SAR data. Novak et al. [15] later showed that the PWF leads to an enhanced target detection performance. The PWF was further developed to multi-look polarimetric whitening filter (MPWF) by Lopes and Liu et al. [16]-[17].

In this section, we review the previously proposed statistical models for the MPWF.
No texture: In absence of texture, the speckle is fully developed, and the covariance matrix obeys the Wishart distribution [2]. Given a complex pixel that follow the circular complex Gaussian of dimension the sample covariance matrix follows the scaled complex Wishart distribution [2][25]. The output of the MPWF is as follow [3]

$$
z=\frac{1}{L} \sum_{i=1}^{L} \mathbf{y}_{i}^{\dagger} \Sigma^{-1} \mathbf{y}_{i}=\operatorname{tr}\left(\Gamma^{-1} \mathbf{Y}\right)
$$

where $\operatorname{tr}(\bullet)$ is the trace operator and $L$ as the number of samples that are independent.

According to literature [27], $z$ obeys a Gamma distribution:

$$
z \sim \gamma\left(L d, \frac{1}{L}\right)
$$

where $\gamma(\alpha, \beta)$ denotes a Gamma distribution with shape parameter $\alpha$, and scale parameter $\beta . \gamma(\alpha, \beta)$ can be represented as [27]

$$
\gamma(\alpha, \beta)=\frac{1}{\Gamma(\alpha)} \frac{1}{\beta}\left(\frac{x}{\beta}\right)^{\alpha-1} e^{-\frac{x}{\beta}}
$$

Texture: if we assume the framework of the product model, when the speckle is partially developed, the output after a polarimetric whitening filter is defined as follows [27]

$$
\begin{aligned}
& z=\frac{1}{L} \sum_{i=1}^{L} \mathbf{k}_{i}^{\dagger} \Sigma^{-1} \mathbf{k}_{i}=\operatorname{tr}\left(\Sigma^{-1} \mathbf{C}\right) \\
& =\frac{\tau}{\mathrm{E}\{\tau\}} \operatorname{tr}\left(\boldsymbol{\Gamma}^{-1} \mathbf{Y}\right)=\tilde{\tau} x
\end{aligned}
$$

where $\tilde{\tau}$ is the unitary texture variable and $x=\operatorname{tr}\left(\Gamma^{-1} \mathbf{Y}\right)$. Eq (9) and $\mathrm{Eq}(10)$ turn to be the single look complex (SLC) case when $L=1$. Except for the K-Wishart distribution case, the statistics of $z$ is unknown under different texture models. The derivations of the PDFs of $z$ for different texture models are presented in section III and are part of the novel contribution of this work.

\section{The Probability of FAlSe Alarm IN DIFFERENT STATISTICAL MODELS}

In this section, we derive the PDF and PFA of images after the filters PWF or MPWF are applied. We consider product models which cover the whole log-cumulants plane. The different cases are considered in the following.

\section{A. Wishart Distribution Case}

Eq. (9) shows the statistics of the MPWF in Wishart distribution case (no texture) [27]. In this statistical model, the PFA can be obtained from Appendix A:

$$
P_{f a}=\frac{\Gamma(d L, L T)}{\Gamma(d L)}
$$

where $P_{f a}$ is the false alarm rate, $d$ is the dimension of the scattering vector. The gamma function and the incomplete gamma function are defined as follows[28]

$$
\Gamma(x)=\int_{0}^{\infty} e^{-t} t^{x-1} d t, \Gamma(a, x)=\int_{x}^{\infty} e^{-t} t^{a-1} d t
$$

Therefore, the threshold $T$ in the CFAR process can be derived when the false alarm rate (FAR) is $P_{f a}$ 


$$
T=\frac{\Gamma^{-1}\left(d L, P_{f a} \Gamma(d L)\right)}{L}
$$

where $\Gamma^{-1}(\bullet)$ is the inverse function of the incomplete Gamma function [28]. It can be seen the PFA and the threshold $T$ can be calculated even if $L$ is a non-integer number. The existed expression of PFA when $L$ is an integer was derived in [3]

$$
P_{f a}=e^{-L T} \sum_{k=0}^{d L-1} \frac{(L T)^{k}}{k !}
$$

We should justify whether they are the same. Since the Gamma function can be expressed as follows when $L$ is an integer [28]

$$
\begin{gathered}
\Gamma(L+1, z)=L ! e^{-z} \sum_{k=0}^{L} \frac{z^{k}}{k !} \\
\Gamma(L+1)=L !
\end{gathered}
$$

Substitute Eq. (16-17) into Eq. (12), it can be obtained

$$
P_{f a}=\frac{\Gamma(d L, L T)}{\Gamma(d L)}=e^{-L T} \sum_{k=0}^{d L-1} \frac{(L T)^{k}}{k !}
$$

where $\Gamma(\bullet)$ is the standard gamma function. It can be seen that the new form of the PFA in Eq. (12) is an extension of the existing result in Eq. (15), while the Eq. (15) can only be used in the integer case.

\section{B. $K$-Wishart Distribution Case}

If the texture variable $\tilde{\tau}$ obeys a Gamma distribution, the multi-look covariance matrix $\mathbf{C}$ obeys the K-Wishart distribution. The textural variable $\tilde{\tau}$ obeys the unitary Gamma distribution as [27]

$$
\tilde{\tau} \sim \gamma\left(\alpha, \frac{1}{\alpha}\right)=\frac{\alpha^{\alpha}}{\Gamma(\alpha)} \tilde{\tau}^{\alpha-1} e^{-\alpha \tilde{\tau}}
$$

where $\alpha$ is the shape parameter.

The PDF and the PFA can be obtained from Appendix B. The PDF can be presented as follows:

$$
f_{K}(z ; \alpha, L, d)=\frac{2(\alpha L)^{\frac{\alpha+L d}{2}} z^{\frac{\alpha+L d}{2}-1}}{\Gamma(L d) \Gamma(\alpha)} K_{\alpha-L d}\left[2(\alpha L z)^{1 / 2}\right]
$$

where $K_{\alpha-L d}(\cdot)$ is the second type of modified Bessel function, which is defined as [29]

$$
K_{v}(z)=\frac{\Gamma(v+1 / 2)(2 z)^{v}}{\sqrt{\pi}} \int_{0}^{\infty} \frac{\cos u}{\left(u^{2}+z^{2}\right)^{v+1 / 2}} d u
$$

The PFA can be derived as follows when the threshold is $T$ in the CFAR process

$$
P_{f a}=1-\frac{1}{\Gamma(L d) \Gamma(\alpha)} G_{1,3}^{2,1}\left[\alpha L T \mid \begin{array}{c}
1 \\
\alpha, L d, 0
\end{array}\right]
$$

where $G(\bullet)$ is Meijer $\mathrm{G}$ function [30]

$$
\begin{aligned}
& G_{p, q}^{m, n}\left[z\left[\begin{array}{l}
z \\
a_{1}, \cdots, a_{p} \\
b_{1}, \cdots, b_{q}
\end{array}\right]=\frac{1}{2 \pi i}\right. \\
& \times \int_{L} \frac{\prod_{j=1}^{m} \Gamma\left(b_{j}-s\right) \prod_{j=1}^{n} \Gamma\left(1-a_{j}+s\right)}{\prod_{j=m+1}^{q} \Gamma\left(1-b_{j}+s\right) \prod_{j=n+1}^{p} \Gamma\left(a_{j}-s\right)} z^{s} d s
\end{aligned}
$$

In literature [22] the PFA has also been derived using a characteristic function. This is complicated and can only be used when the ENL is an integer.

\section{G-Wishart Distribution Case}

If the texture variable $\tilde{\tau}$ obeys the inverse Gamma distribution, the multi-look covariance matrix $\mathbf{C}$ obeys the G0-Wishart distribution. The unitary variable $\tilde{\tau}$ obeys the unitary inverse Gamma distribution as [7]

$$
f(\tilde{\tau} ; \lambda)=\frac{(\lambda-1)^{\lambda}}{\Gamma(\lambda)} \tilde{\tau}^{-\lambda-1} \exp \left(\frac{1-\lambda}{\tilde{\tau}}\right) \quad(\lambda, \tilde{\tau}>0)
$$

where $\lambda$ is the shape parameter.

The PDF and the PFA can be obtained from Appendix C. The PDF is presented as follows:

$$
f_{G 0}(z ; \lambda, L, d)=\frac{L^{L d} z^{L d-1}(\lambda-1)^{\lambda}}{B(L d, \lambda)(\lambda+L z-1)^{L d+\lambda}}
$$

where $B(a, b)$ is the Beta function[28]

$$
B(a, b)=\frac{\Gamma(a) \Gamma(b)}{\Gamma(a+b)}
$$

If the threshold is $T$, the PFA is

$$
\begin{aligned}
& P_{f a}=1-\frac{1}{B(L d, \lambda) L d}\left(\frac{L T}{\lambda-1}\right)^{L d} \\
& \times_{2} F_{1}\left(L d+\lambda, L d ; 1+L d ;-\frac{L T}{\lambda-1}\right)
\end{aligned}
$$

where ${ }_{2} F_{1}(\cdot)$ is the Hyper geometric function[28], and $B(a, b)$ is the Beta function.

\section{Fisher Wishart Distribution Case}

If the texture variable $\tilde{\tau}$ obeys a Fisher distribution, the multilook covariance matrix $\mathbf{C}$ will obey a Fisher Wishart (F-Wishart) distribution. The unitary variable $\tilde{\tau}$ obeys the unitary Fisher distribution as [23]

$$
\tilde{\tau} \sim f(\tilde{\tau} ; u, v)=\frac{1}{B(u, v)} \frac{\omega(\omega \tilde{\tau})^{v-1}}{(\omega \tilde{\tau}+1)^{u+v}}
$$

where $u$ and $v$ are the freedom parameters, $\Gamma(\cdot)$ is the Gamma function, and $\omega=v /(u-1)$.

The PDF can be obtained from Appendix D.

$$
\begin{aligned}
& f_{F}(z ; u, v, L, d)=\frac{[L z \omega]^{L d}}{z \Gamma(L d)} \frac{1}{B(u, v)} \\
& \times \Gamma(u+L d) U[u+L d, L d+1-v, L z \omega]
\end{aligned}
$$

where $U(a, b, z)$ is the KummerU function[28]

$$
U(a, b, z)=\frac{1}{\Gamma(a)} \int_{0}^{+\infty} \exp (-z t) t^{a-1}(1+t)^{b-a-1} d t
$$

If the threshold is $T$, the PFA is (Appendix D)

$$
\begin{aligned}
& P_{f a}=1-\frac{[L T \omega]^{L d+1} \Gamma(L d+1)}{\Gamma^{2}(L d) \Gamma(u) \Gamma(v) L d} \\
& \times G_{2,3}^{2,2}\left(\begin{array}{l}
\left.L T \omega \mid \begin{array}{l}
-L d-u,-L d \\
v-L d-1,-1,-L d-1
\end{array}\right)
\end{array}\right.
\end{aligned}
$$


where $G(\bullet)$ is Meijer G function, $\Gamma(\bullet)$ is the standard gamma function and $\omega=v /(u-1)$, which is the same definition in Eq (28).

\section{E. Inverse Beta Wishart Case}

If the texture obeys $\tilde{\tau}$ an inverse beta distribution the multi-look covariance matrix $\mathbf{C}$ obeys an inverse beta Wishart (IB-Wishart) distribution. The unitary variable $\tilde{\tau}$ obeys the unitary inverse Beta distribution as [23]

$$
\begin{aligned}
& \tilde{\tau} \sim f(\tilde{\tau} ; u, v)=\frac{1}{B(u, v)} \\
& \times \eta(\eta \tilde{\tau}-1)^{u-1}(\eta \tilde{\tau})^{-u-v}, \quad \tilde{\tau} \in\left[\frac{1}{\eta}, \infty\right]
\end{aligned}
$$

where $\eta=(u+v-1) /(v-1)$, and $u, v$ are the freedom parameters of the inverse beta distribution.

The PDF and the PFA can be obtained from Appendix E. The $\mathrm{PDF}$ is presented as follows

$$
\begin{aligned}
& f_{I B}(z ; u, v, L, d)=\frac{[L z \eta]^{L d}}{z B(L d, v)} \frac{\Gamma(u+v)}{\Gamma(L d+v+u)} \\
& \times M(L d+v, L d+v+u,-L z \eta)
\end{aligned}
$$

where $M(a, b, z)$ is the KummerM function [28]

$$
M(a, b, z) \Gamma(a) \frac{\Gamma(b-a)}{\Gamma(b)}=\int_{0}^{1} \exp (z t)(1-t)^{b-a-1} t^{a-1} d t
$$

If the threshold is $T$, the PFA is

$$
\begin{aligned}
& P_{f a}=1-\frac{[L T \eta]^{L d+1} \Gamma(L d+1) \Gamma(v+u)}{\Gamma^{2}(L d) \Gamma(v) L d} \times \\
& G_{2,3}^{1,2}\left(L T \eta \mid \begin{array}{l}
-L d-v,-L d \\
-1,-L d-1,-L d-u-v
\end{array}\right)
\end{aligned}
$$

where $\Gamma(\bullet)$ is the standard gamma function, $G(\bullet)$ is Meijer $\mathrm{G}$ function, and $\eta=(u+v-1) /(v-1)$, which is the same definition in $\mathrm{Eq}(32)$.

\section{F. Beta Wishart Distribution Case}

If the texture variable $\tilde{\tau}$ obeys a beta distribution, the multi-look covariance matrix $\mathbf{C}$ obeys the Beta Wishart (B-Wishart) distribution. The unitary variable $\tilde{\tau}$ obeys the unitary Beta distribution as [23]

$$
\begin{aligned}
& \tilde{\tau} \sim f(\tilde{\tau} ; u, v)=\frac{1}{B(u, v)} \\
& \times \xi(\xi \tilde{\tau})^{v-1}(1-\xi \tilde{\tau})^{u-1}, \quad \tilde{\tau} \in\left[0, \frac{1}{\xi}\right]
\end{aligned}
$$

where $\xi=v /(u+v)$, and $u, v$ are the freedom parameters of beta distribution.

The PDF and the PFA can be obtained from Appendix F. The $\mathrm{PDF}$ is presented as follows.

$$
\begin{aligned}
& f_{B}(z ; u, v, L, d)=z^{\frac{L d+v-3}{2}} \frac{[L \xi]^{L d}}{\Gamma(L d)} \frac{1}{B(u, v)} \\
& \times \exp (-L z \xi) \quad \mathrm{W}_{\frac{L d-v-2 u+1}{2}, \frac{L d-v}{2}}(L z \xi)
\end{aligned}
$$

where $\mathrm{W}_{k, \mu}(x)$ is the WhittakerW function [28]

$$
\mathrm{W}_{k, \mu}(x)=x^{\mu+\frac{1}{2}} \exp \left(-\frac{x}{2}\right) U\left(\mu-k+\frac{1}{2}, 2 \mu+1, x\right)
$$

If the threshold is $T$, the PFA is

$$
\begin{aligned}
& P_{f a}=1-\frac{[L T \xi]^{L d+1} \Gamma(L d+1) \Gamma(v+u)}{\Gamma^{2}(L d) \Gamma(v) L d} \\
& \times G_{2,3}^{2,1}\left(L T \xi \mid \begin{array}{l}
-L d, \quad v+u-L d-1 \\
v-L d-1,-1,-L d-1
\end{array}\right)
\end{aligned}
$$

where $\Gamma(\bullet)$ is the standard gamma function, $G(\bullet)$ is Meijer $\mathrm{G}$ function, and $\xi=v /(u+v)$, which is the same definition in Eq (36).

\section{G. H Wishart Distribution Case}

If the texture variable $\tilde{\tau}$ obeys a GГD distribution, the multilook covariance matrix $\mathbf{C}$ obeys the $\mathrm{H}$ Wishart (H-Wishart) distribution. The unitary variable $\tilde{\tau}$ obeys the normalized GГD distribution, whose PDF is [24]

$$
\tilde{\tau} \sim f(\tilde{\tau} ; \kappa, \nu)=\frac{|\nu| \kappa^{\kappa}}{\sigma \Gamma(\kappa)}\left(\frac{\tilde{\tau}}{\sigma}\right)^{\kappa \nu-1} \exp \left\{-\kappa\left(\frac{\tilde{\tau}}{\sigma}\right)^{\nu}\right\}
$$

where $\sigma=\kappa^{1 / \nu} \Gamma(\kappa) / \Gamma(\kappa+1 / \nu)$, and $\sigma, k, \nu$ are the scale, shape and power parameters of GГD distribution.

The PFD and the PFA can be obtained from Appendix G:

$$
\begin{aligned}
& f_{\mathrm{H}}(z ; \kappa, \nu, L, d)=\frac{\kappa^{1 / \nu} L}{\Gamma(L d) \Gamma(\kappa) \sigma} \\
& \begin{cases}\times H_{0,2}^{2,0}\left[\frac{L z \kappa^{1 / \nu}}{\sigma} \mid \begin{array}{cc}
(L d-1,1) & \left(\kappa-\frac{1}{\nu}, \frac{1}{\nu}\right)
\end{array}\right] & v>0 \\
\times H_{1,1}^{1,1}\left[\frac{L z \kappa^{1 / \nu}}{\sigma} \mid \begin{array}{c}
\left(\kappa-\frac{1}{\nu}+1, \frac{1}{\nu}\right) \\
(L d-1,1)
\end{array}\right] & v<0\end{cases}
\end{aligned}
$$

If the threshold is $T$, the PFA should be

$$
\begin{aligned}
& P_{f a}=1-\frac{T \kappa^{1 / \nu} L}{\Gamma(L d) \Gamma(\kappa) \sigma}
\end{aligned}
$$

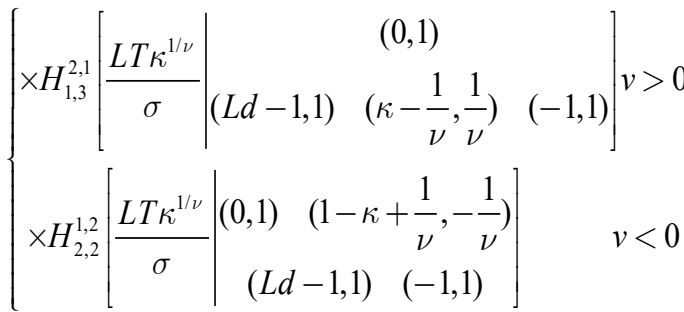

where $P_{f a}$ is the false alarm rate, $T$ is the detection threshold, $L$ is the equivalent number of looks, $d$ is the dimension of the polarimetric scattering vector, $\Gamma(\cdot)$ is the Gamma function and $\sigma, \kappa, \nu$ are the scale, shape and power parameters of GГD distribution.

$H(\cdot)$ is the Fox $\mathrm{H}$-function defined as [31] 


$$
\begin{aligned}
& H_{p, q}^{m, n}\left[z\left[\begin{array}{l}
\left(a_{i}, A_{i}\right)_{1, p} \\
\left(b_{j}, B_{j}\right)_{1, q}
\end{array}\right]=\frac{1}{2 \pi i}\right. \\
& \times \int_{\Theta} \frac{\prod_{j=1}^{m} \Gamma\left(b_{j}+B_{j} s\right) \prod_{j=1}^{n} \Gamma\left(1-a_{j}-A_{i} s\right)}{\prod_{j=n+1}^{p} \Gamma\left(a_{j}+A_{i} s\right) \prod_{j=m+1}^{q} \Gamma\left(1-b_{j}-B_{j} s\right)} z^{-s} d s
\end{aligned}
$$

where $z, c, a_{i}, b_{j} \in \mathbb{C}, A_{i}, B_{j} \in \mathbb{R}^{+}$, and $m, n, p, q \in \mathbb{Z}$ such that $1 \leq j \leq m, 1 \leq i \leq n, 0 \leq m \leq q, o \leq n \leq p, A_{i}>0, B_{j}>0 ; \Theta$ is a contour in the complex s-plane ranging from $\varsigma-i \infty$ to $\varsigma+i \infty$.

\section{NOVEL PARAMETER ESTIMATION METHOD}

CFAR algorithms need to estimate the clutter PDF parameters locally. This estimation has an obvious influence on the detection performance. Traditionally, the second and third log-cumulants of the multi-look covariance matrix are used for the parameter estimation in product models. Recently estimators via log-cumulants on the MPWF were proposed [32] in both $\mathrm{K}$ and $\mathrm{G} 0$ distribution cases. These cannot be used in the single look complex (SLC) data. Additionally, until now these estimators have not been extended to other product models.

\section{A. Novel Estimators for PWF based on log-cumulants}

Here the estimators using MPWF and based on log-cumulants are modified to be suitable for the other product models. The logarithmic transformation translates the product model into an additive one by the Mellin Transform [33]. The $\log$-cumulants of the output of MPWF $z$ in the product model can be expressed as follows [33]

$$
k_{n}\{z\}=k_{n}\{\tilde{\tau}\}+k_{n}\{x\}
$$

where $z$ is the MPWF output, $\tilde{\tau}$ is the unitary textual variable, $x$ the MPWF output as a gamma distribution in Wishart model, and $k_{n}\{\bullet\}$ is the $\mathrm{n}$-th order log-cumulant. $k_{n}\{x\}$ is the $\mathrm{n}$-th order log-cumulant of $x$ which can be derived [34]

$$
\begin{aligned}
& k_{1}\{x\}=\psi^{(0)}(L d)-\ln L \\
& k_{n}\{x\}=\psi^{(n-1)}(L d), \forall n>1
\end{aligned}
$$

Therefore, Eq. (44) can be rewritten as

$$
\begin{aligned}
& k_{1}\{z\}=\psi^{(0)}(L d)-\log L+k_{1}\{T\} \\
& k_{n}\{z\}=\psi^{(n-1)}(L d)+k_{n}\{T\}, \forall n>1
\end{aligned}
$$

where $\Gamma(\bullet)$ is the standard Euler gamma function, and $\psi^{(m)}(z)$ is defined as[33]

$$
\psi^{(m)}(z)=\frac{d^{m+1}}{d z^{m+1}} \ln \Gamma(L)=(-1)^{m} \int_{0}^{\infty} \frac{t^{m} e^{-z t}}{1-e^{-t}} d t
$$

The log-cumulants of the scaled texture can be found in [24][34]. They are also listed in Table I. The first, second and

\begin{tabular}{|c|c|}
\hline Texture distribution & Log-cumulants \\
\hline \multirow{2}{*}{ Gamma } & $k_{1}=\psi^{(0)}(\alpha)-\ln \alpha$ \\
\hline & $k_{n}=\psi^{(n-1)}(\alpha), \quad n>1$ \\
\hline \multirow{2}{*}{ Inverse Gamma } & $k_{1}=-\psi^{(0)}(\lambda)+\ln (\lambda-1)$ \\
\hline & $k_{n}=(-1)^{n} \psi^{(n-1)}(\lambda), \quad n>1$ \\
\hline \multirow{4}{*}{ Fisher } & $k_{1}=\psi^{(0)}(v)-\ln v$ \\
\hline & $-\psi^{(0)}(u)+\ln (u-1)$ \\
\hline & $k_{n}=\psi^{(n-1)}(v)$ \\
\hline & $+(-1)^{n} \psi^{(n-1)}(u), n>1$ \\
\hline \multirow{4}{*}{ Beta } & $k_{1}=\psi^{(0)}(v)-\ln v$ \\
\hline & $-\psi^{(0)}(u+v)+\ln (u+v)$ \\
\hline & $k_{n}=\psi^{(n-1)}(v)$ \\
\hline & $-\psi^{(n-1)}(u+v), n>1$ \\
\hline \multirow{4}{*}{ Inverse Beta } & $k_{1}=-\psi^{(0)}(v)+\ln (v-1)$ \\
\hline & $+\psi^{(0)}(u+v)-\ln (u+v-1)$ \\
\hline & $k_{n}=(-1)^{n}\left[\psi^{(n-1)}(v)\right.$ \\
\hline & $\left.-\psi^{(n-1)}(u+v)\right], n>1$ \\
\hline \multirow{3}{*}{ GГD } & $k=\frac{\Psi(0, k)}{\ln \int \quad \Gamma(k)}$ \\
\hline & $v \quad(\Gamma(k+1 / v))$ \\
\hline & $k_{n}=\frac{\Psi(n-1, k)}{v^{n}}$ \\
\hline
\end{tabular}
third order log-cumulants of the multilook covariance matrix can be used to estimate the shape parameters in different distributions. The novel estimators may improve the accuracy when using MPWF, as it has been proven with the K and G0 distribution cases [32].

TABLE I

LOG-CUMULANTS OF DIFFERENT TEXTURE DISTRIBUTIONS

\section{B. Comparisons of Different Parameter Estimators}

In this section we compare the following three estimators: a) the method via second and third order log-cumulants of the covariance matrix $(k 2 k 3), \mathrm{b})$ the first and second order log-cumulants after an MPWF ( $k 1 k 2-\mathrm{MPWF})$ and c) the second and third order log-cumulants after an MPWF ( $k 2 k 3$-MPWF).

Monte Carlo simulations based on the product model are used to produce the simulated SAR data. We generated the Wishart distribution covariance matrix and the textual variable. The Wishart part of the synthetic data set consists of $N$ $=1,000,000$ covariance matrix samples drawn from a complex, circular, and zero-mean Wishart distribution. The distribution was modulated by a scale matrix $\Gamma$ that is computed by averaging a homogeneous region in the measured SAR data. The number of looks was set to $L=10$. The textual variable is generated according to the distribution parameters in Table II. 
TABLE II

SIMULATION PARAMETERS OF DIFFERENT TEXTURES

\begin{tabular}{ccccc} 
& Fisher & Beta & IBeta & GГD \\
\hline$u(\kappa)$ & 5 & 2 & 2 & 2 \\
\hline$v(\nu)$ & 8 & 3 & 3 & 0.5
\end{tabular}

From $N=1,000,000$ product model samples, we drew $M_{b}=$ 10,000 bootstrap samples of variable size $N_{b}$ ([ [ $\begin{array}{llll}16 & 32 & 64 & 128\end{array}$ $2565121024])$ and then used the bootstrap estimator [25] to estimate the bias, variance (Var) and mean square error (MSE) of the estimators.

For an arbitrary parameter to be estimated $\beta$, the Bias, Var and MSE are defined as relative errors [32]:

$$
\begin{aligned}
& \operatorname{Bias}(\hat{\beta})=|\mathrm{E}(\hat{\beta})-\beta| / \beta \\
& \operatorname{Var}(\hat{\beta})=\mathrm{E}\left\{[\mathrm{E}(\hat{\beta})-\hat{\beta}]^{2}\right\} / \beta^{2} \\
& \operatorname{MSE}(\hat{\beta})=\mathrm{E}\left[(\hat{\beta}-\beta)^{2}\right] / \beta^{2}
\end{aligned}
$$

where $\beta$ is the true value of the parameter, $\hat{\beta}$ is the estimated value, and $\mathrm{E}(\bullet)$ is the expect operator. These indexes are used to assess the performances of different estimators.

In the F-Wishart distribution case, the estimation results by different methods are presented in Fig 2. It can be seen that the $k 2 k 3$-MPWF gives the best performance with the least bias, variance, and MSE in the estimation of $u$ and $v$, especially when the sample number is larger than 256.

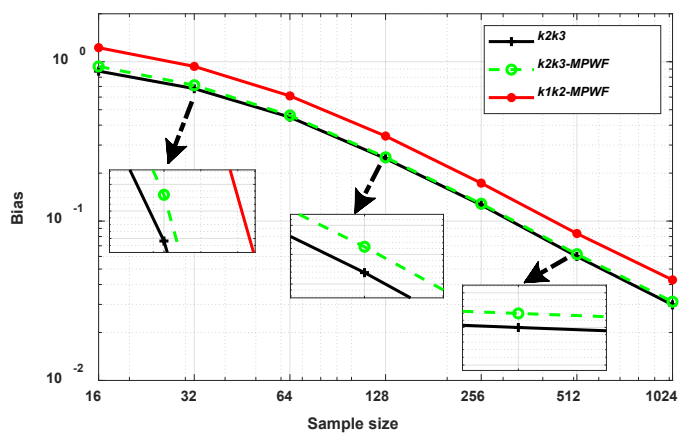

(a) Bias of $u$ estimation

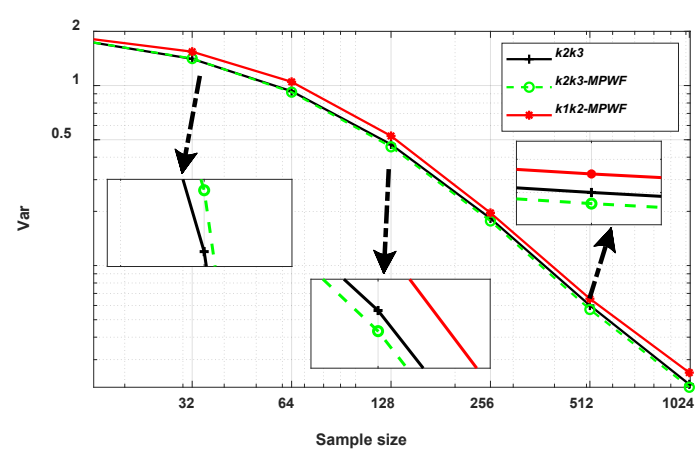

(b) Variance of $u$ estimation

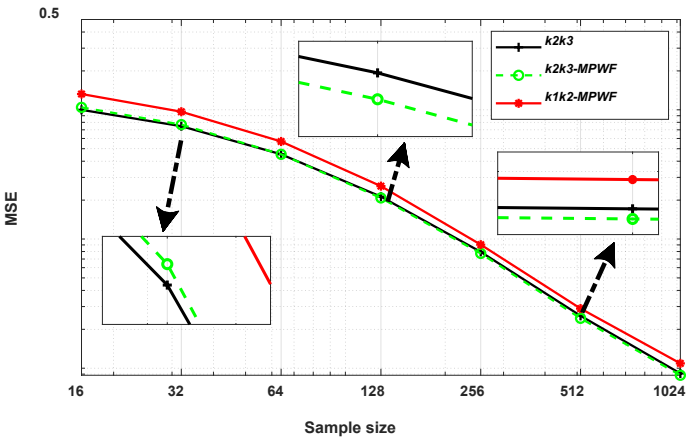

(c) MSE of $u$ estimation

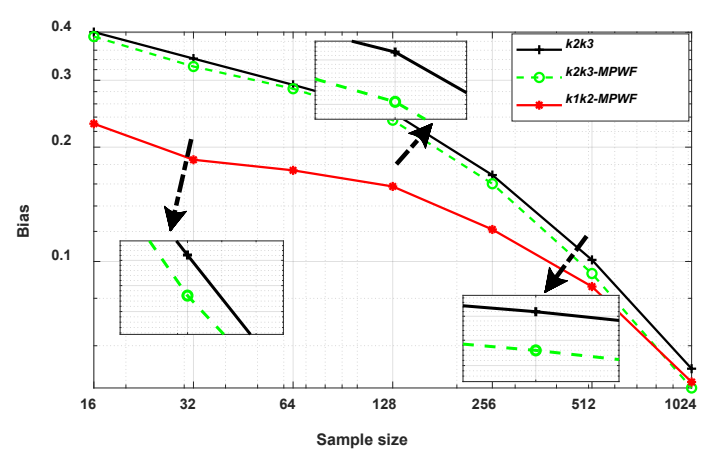

(d) Bias of $v$ estimation

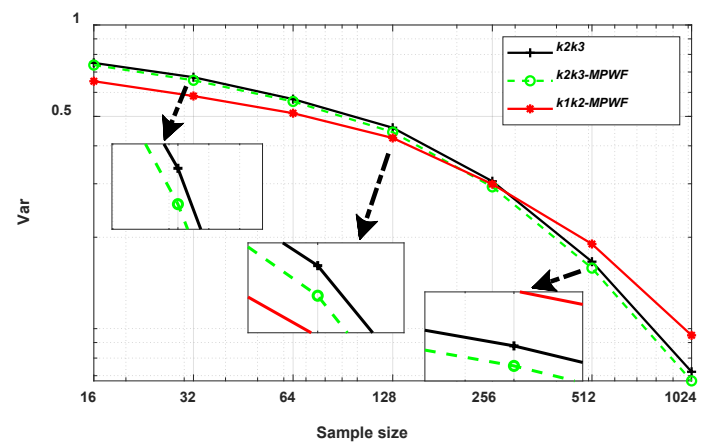

(e) Variance of $v$ estimation

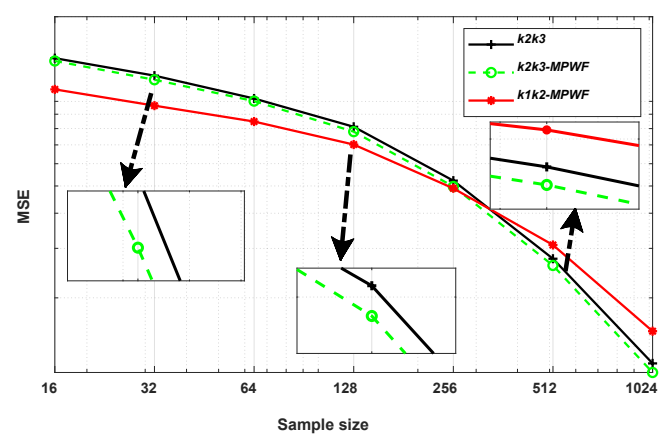

(f) MSE of $v$ estimation

Fig. 2. The result comparison of different estimators in the F-Wishart case.

In the B-Wishart distribution case, the estimation results by different methods are presented in Fig 3. It can be seen that the k1k2-MPWF gives the best performance with the least bias, variance, and MSE. The estimator of $\mathrm{k} 2 \mathrm{k} 3$ and the estimator of $\mathrm{k} 2 \mathrm{k} 3-\mathrm{MPWF}$ almost have the same performance. 


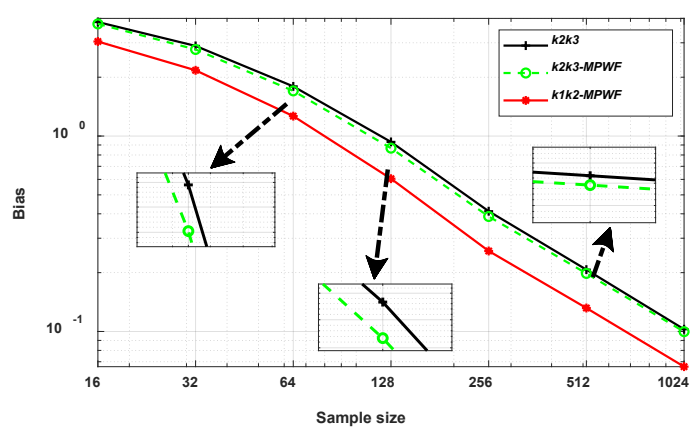

(a) Bias of $u$ estimation

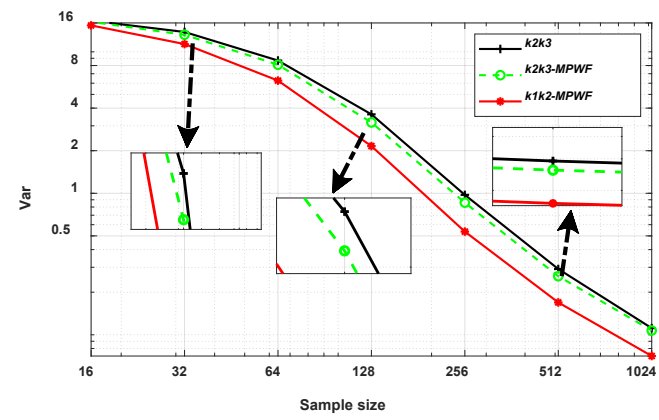

(b) Variance of $u$ estimation

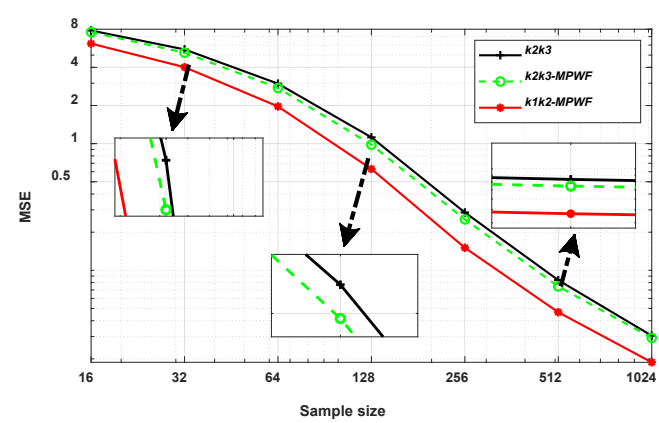

(c) MSE of $u$ estimation

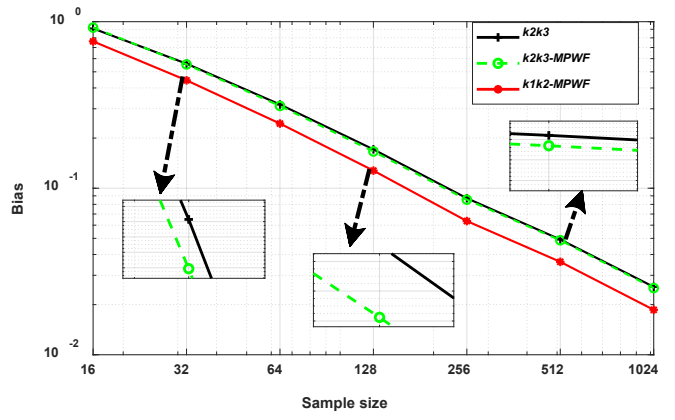

(d) Bias of v estimation

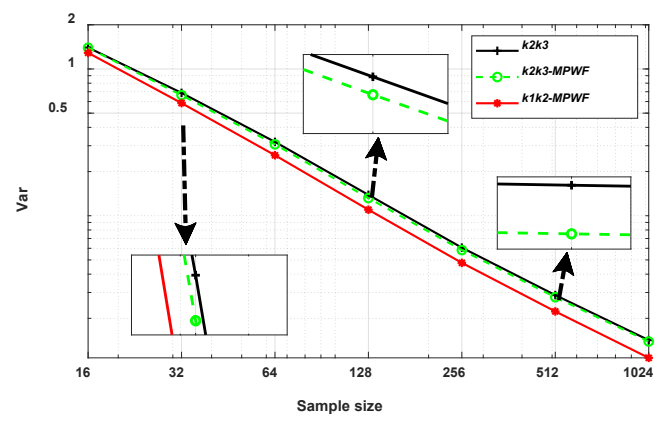

(e) Variance of $v$ estimation

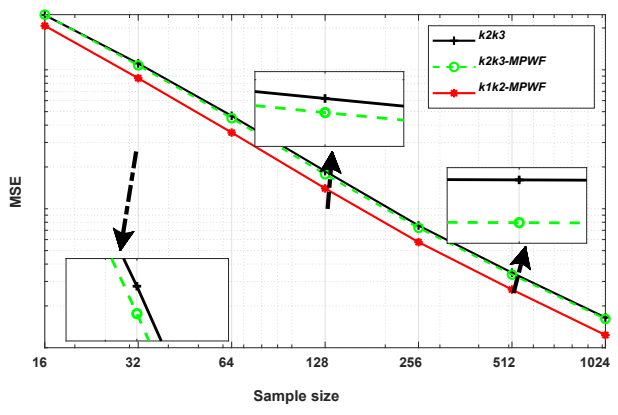

(f) MSE of $v$ estimation

Fig. 3. The result comparison of different estimators in the B-Wishart case.

In the IB-Wishart distribution case, the estimation results by different methods are presented in Fig 4. It can be seen that the estimators of $\mathrm{k} 2 \mathrm{k} 3$ and $\mathrm{k} 2 \mathrm{k} 3 \mathrm{-MPWF}$ give a better performance with the least bias, variance, and MSE compared with the $\mathrm{k} 1 \mathrm{k} 2-\mathrm{MPWF}$ estimator. The estimator of $\mathrm{k} 2 \mathrm{k} 3$ and the estimator of k2k3-MPWF almost have the same performance.

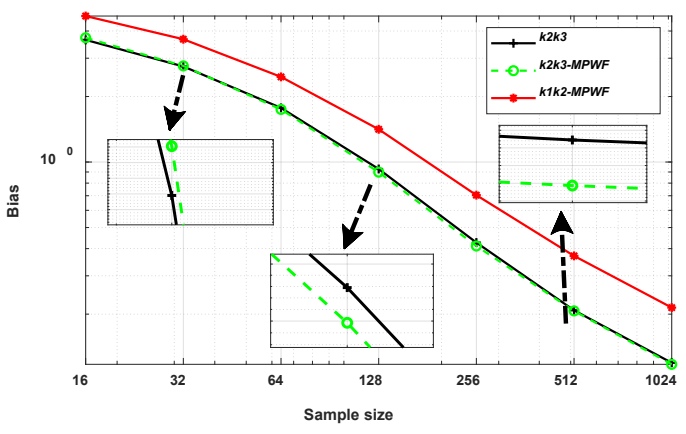

(a) Bias of u estimation

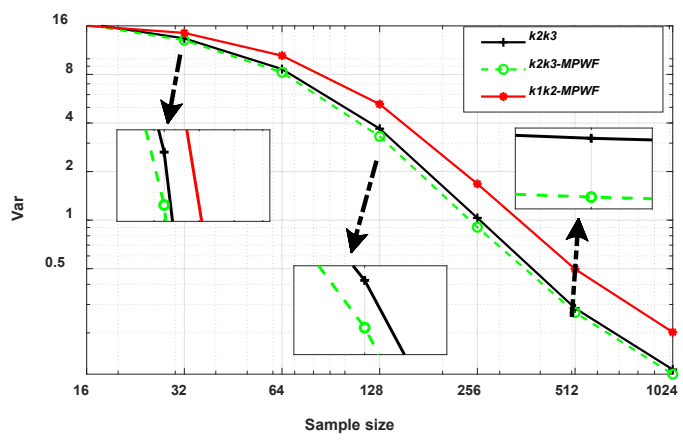

(b) Variance of $u$ estimation

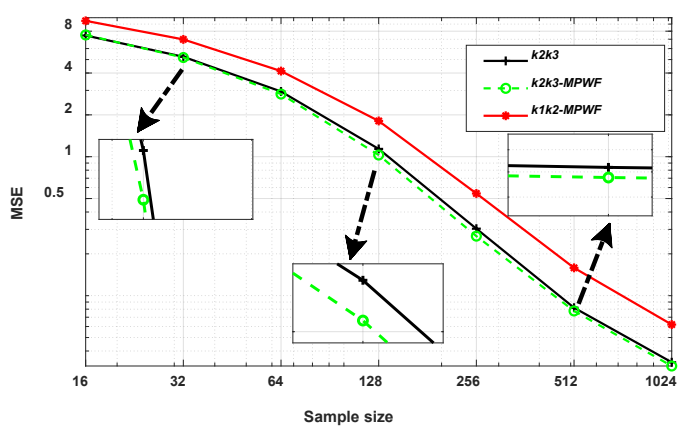

(c) MSE of u estimation 


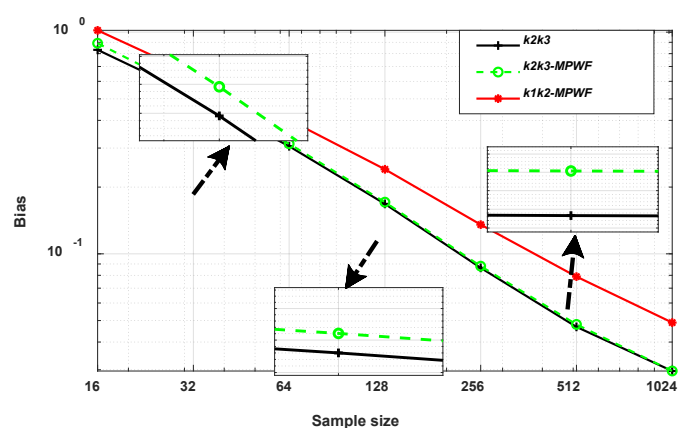

(d) Bias of v estimation

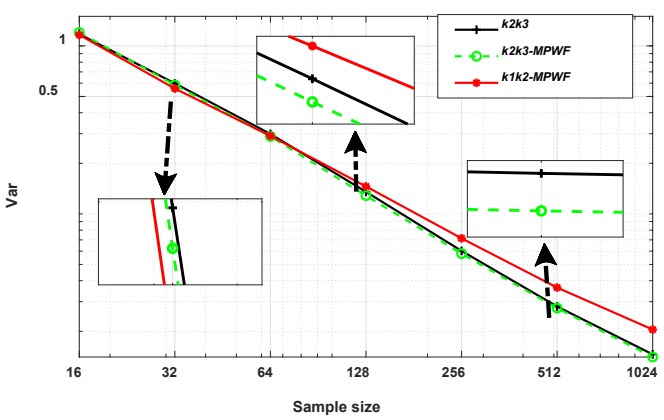

(e) Variance of $v$ estimation

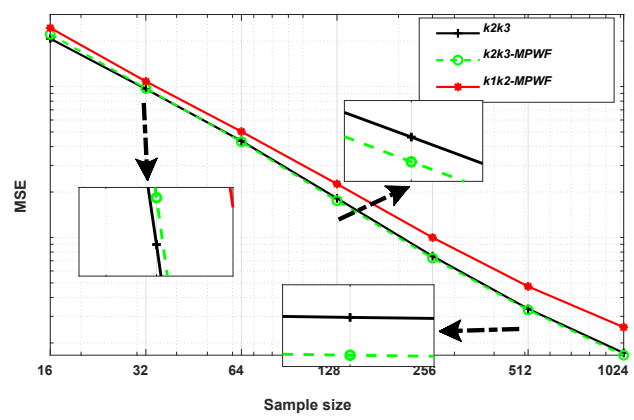

(f) MSE of v estimation

Fig. 4. The result comparison of different estimators in the IB-Wishart case.

In the GГD texture case, it is difficult to perform the numeric calculation for $\mathrm{k} / \mathrm{k} 2-\mathrm{MPWF}$, while the $k 2 k 3$ and the ( $k 2 k 3-\mathrm{MPWF})$ may give a fast estimation for its particular structure in $k_{n}, n>1$ [24]. From Fig 5 it can be seen that the estimators $k 2 k 3$-MPWF give overall the best performance for $\kappa$, and the best performance for $\nu$ when the sample size is large than 512. When the sample size is small, there may be invalid solution for the $k 2-k 3$ equations[35], which leads no monotonicity in Fig 5(d-f) since the invalid results are excluded from the evaluation.

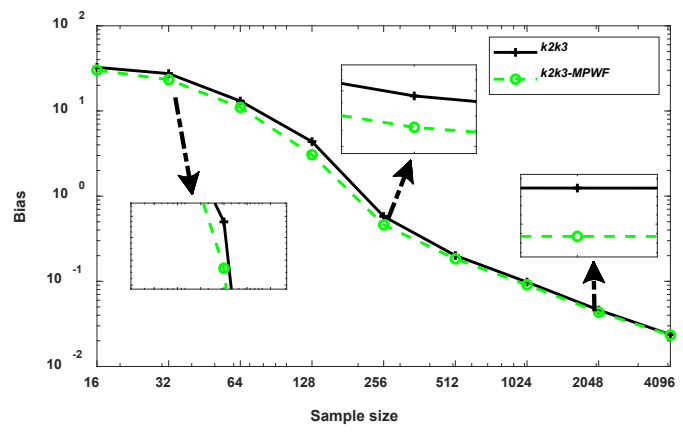

(a) Bias of $k$ estimation

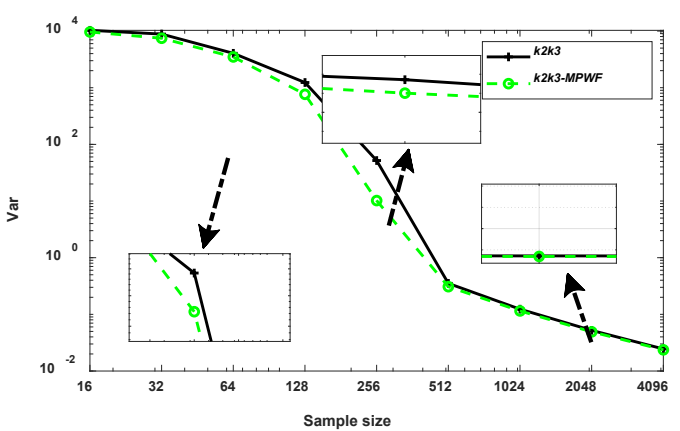

(b) Variance of $k$ estimation

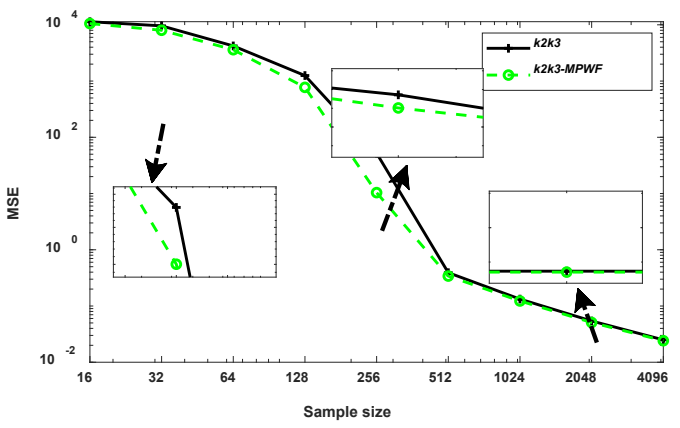

(c) MSE of $k$ estimation

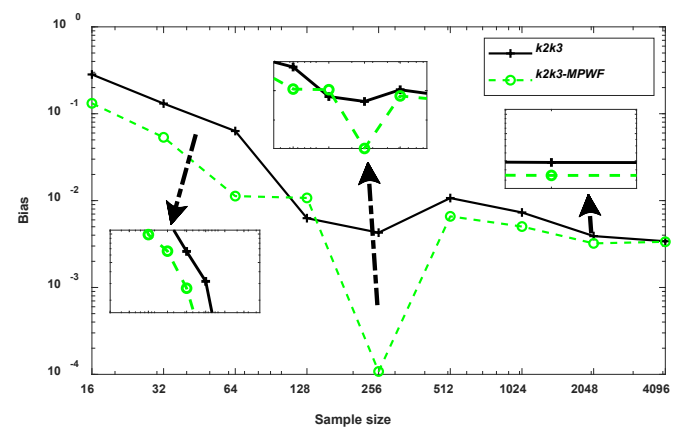

(d) Bias of v estimation

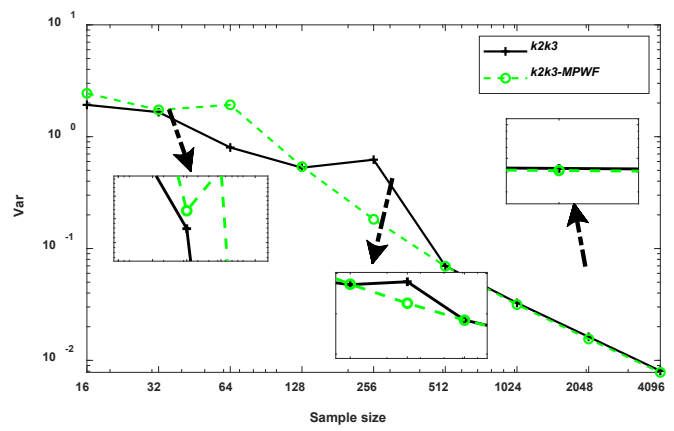

(e) Variance of $v$ estimation

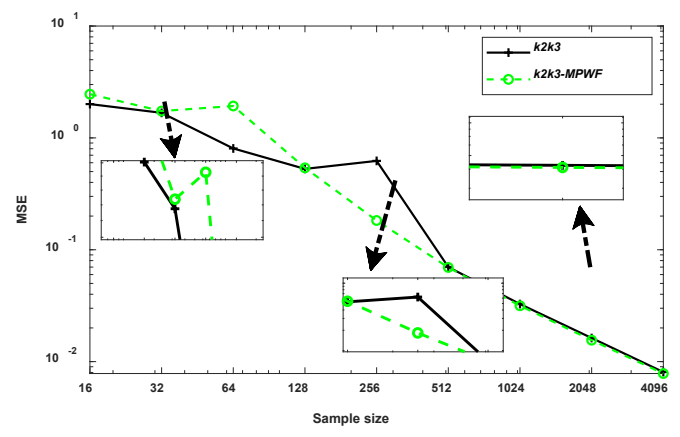

(f) MSE of v estimation

Fig. 5. The result comparison of different estimators in the H-Wishart case. 
Based on the results we should choose the first log-cumulants estimator after an MPWF in the K-Wishart and G0-Wishart [32], the $k 1 k 2$-MPWF estimator, in the B-Wishart distribution case, and the $k 2 k 3-\mathrm{MPWF}$ in the F-Wishart, IB-Wishart and the H-Wishart cases. And from all the figures it can be seen that the performances of $k 2 k 3$ method and $k 2 k 3$-MPWF method have the similar estimation results compared by the MSE especially when the sample size is large.

\section{EXPERIMENTS AND VALIDATION}

\section{A. Simulated data}

Simulated and measured data are used to assess the validity of the CFAR algorithms. The real data is a 9-look NASA/JPL AIRSAR polarimetric dataset that covers an area of the inland sea named Kojimawan close to Tamano City, Japan [36]. The dataset consisting of fully polarimetric data acquired on October 4, 2000 using the AIRSAR instrument onboard a DC-8 aircraft during the PACRIM-2 mission. The range pixel spacing is 3.3 meters, the azimuth pixel spacing is 4.6 meters, the number of range looks (i.e. averaged pixels) is 1 and the number of azimuth looks is 9 .

We generated simulated data from these real data by applying parameter estimation of the statistical models. Here six real sub-images (shown in Fig. 9) are selected to be used as the test areas. Each sub-image is corresponding to the simulated data. The statistical parameters can be estimated by applying parameter estimation methods for a particular statistical model. Monte Carlo simulations based on the product model for each statistical model are then used to produce the simulated data. Therefore, the polarimetric covariance matrices and textual parameters of different clutters are obtained from real data.

To avoid possible contamination in the assessment of the CFAR performance, pure clutter data without ships will be used. We removed ships using rectangles and ships were confirmed by visual inspection of both $\mathrm{C}$ band and $\mathrm{L}$ band images [36].

The purpose of the following experiments is to test how accurate is our analytical solution for the PFA when the data obey a specific distribution. Since we want to have a fair comparison between models we decided not to rely on different real data (since clutter conditions will be different) but use simulated data. In this way we can assure that each parameter stay the same except for the product model used for simulations.

\section{B. Comparing models}

Since several of the models presented in this manuscript are generalizations of others, we only compared classes of models among each other. This provides a clearer visualization of the results.

The H-Wishart is compared with all other models since $\mathrm{H}-\mathrm{Wishart}$ distribution covers many kinds of distributions in the $k 2-k 3 \log$-cumulants plane.

Additionally, in the Wishart distribution case, 2P-CFAR method is the other one adopted and compared, since Wishart distribution is a special case of any other product model.

Fig 1 shows that the K-distribution is a special case of Beta and Fisher distributions, and G0-distribution is a special case of Fisher and Inverse Beta distribution. Therefore, the B-Wishart model is compared with $\mathrm{K}-\mathrm{Wishart,} \mathrm{G0-Wishart,} \mathrm{Wishart} \mathrm{and}$
2P-CFAR models; K-Wishart is compared with G0-Wishart, Wishart and 2P-CFAR models; G0-Wishart is compared with $\mathrm{K}$-Wishart, Wishart and 2P-CFAR models; F-Wishart is compared with K-Wishart, G0-Wishart, Wishart and 2P-CFAR models; IB-Wishart is compared with G0-Wishart, 2P-CFAR, $\mathrm{K}-\mathrm{Wishart}$ and Wishart models.

\section{Results of comparison}

Considering that a direct way to illustrate the CFAR maintenance performance of a model is to provide the PFA of simulated data and the selected model under each detection threshold in its value range, we plotted the curves of PFA against $T$, as shown in Fig 6.

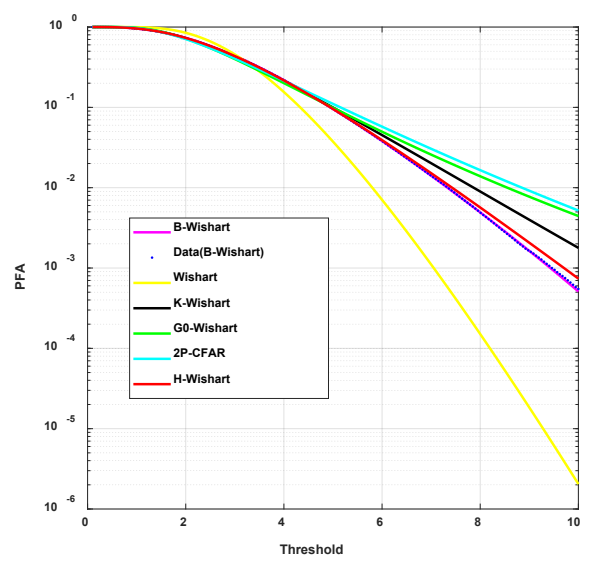

(a) PFAs simulating the data with a B-Wishart model

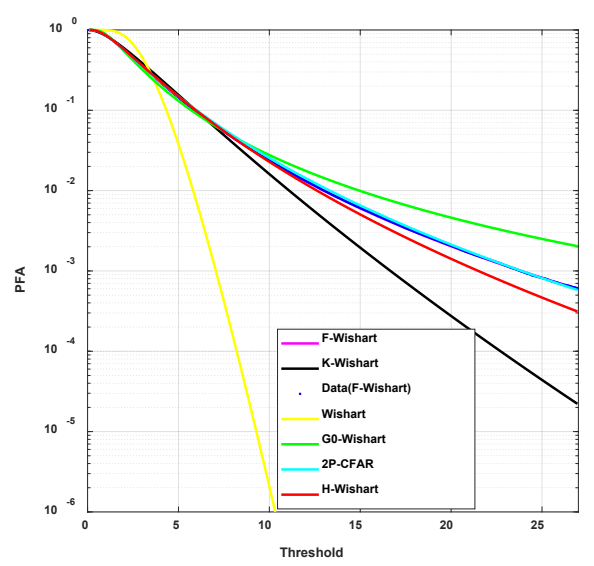

(b) PFAs simulating the data with a F-Wishart model

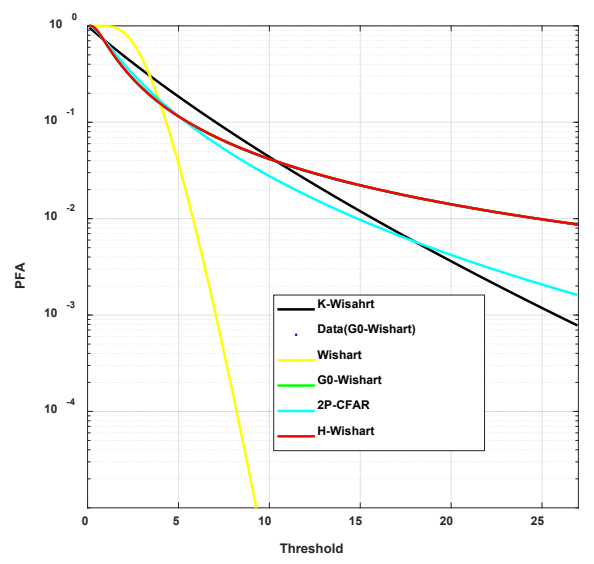

(c) PFAs simulating the data with a G0-Wishart model 


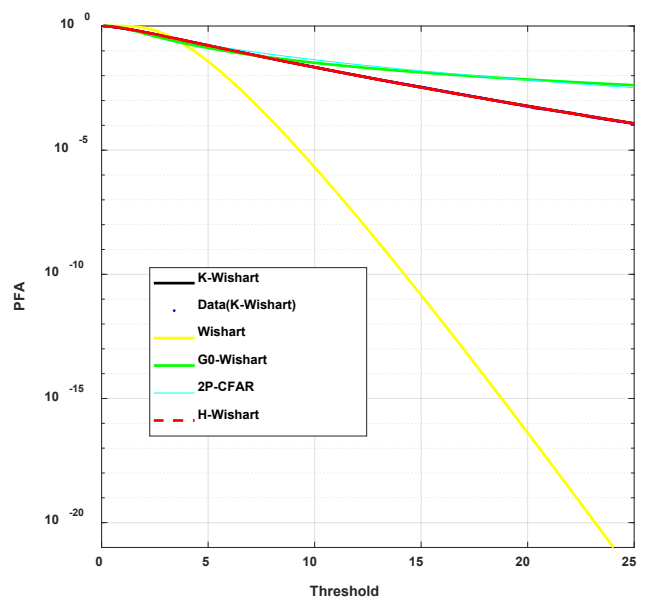

(d) PFAs simulating the data with a K-Wishart model

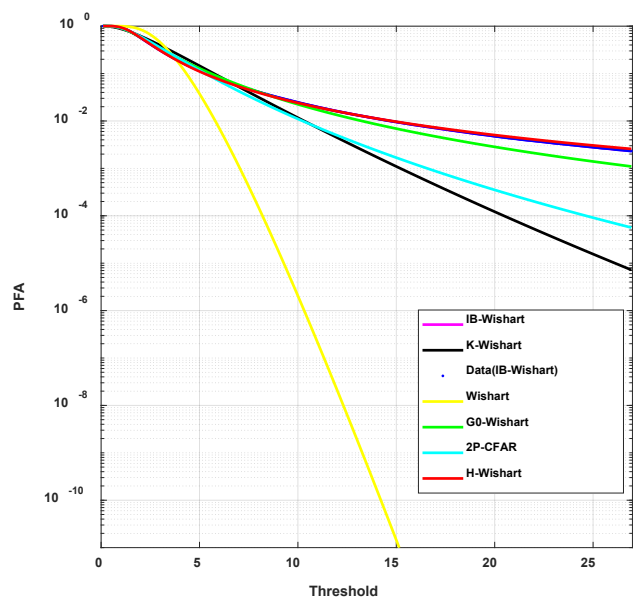

(e) PFAs simulating the data with a IB-Wishart model

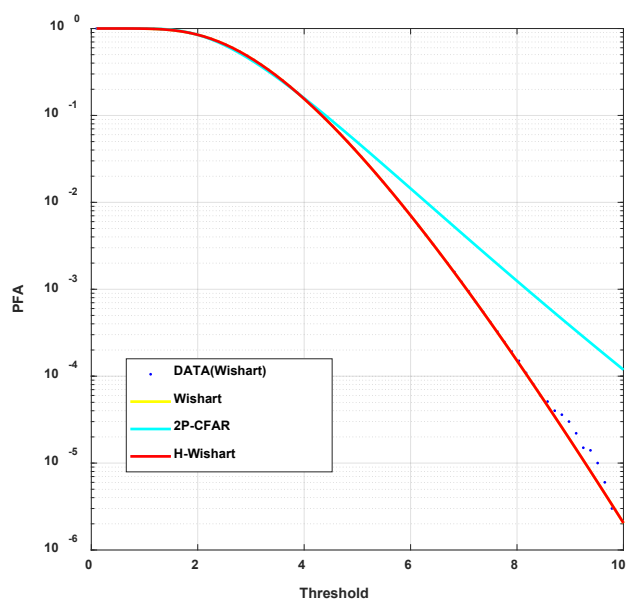

(f) PFAs simulating the data with a Wishart model

Fig. 6. The PFAs in different Statistical models using different CFAR algorithms

To further quantitatively evaluate the CFAR performance of a model, a metric indicating CFAR loss can be defined in decibel.

$$
C_{L}=\left|20 \log \left(\bar{P}_{f a} / \widehat{P}_{f a}\right)\right|
$$

$C_{L}$ is a function dependent on the threshold and indicates the corresponding error between the actual PFA $\bar{P}_{f a}$ and PFA $\widehat{P}_{f a}$ estimated by the model. The $C_{L}$ curves are presented in Fig 7.

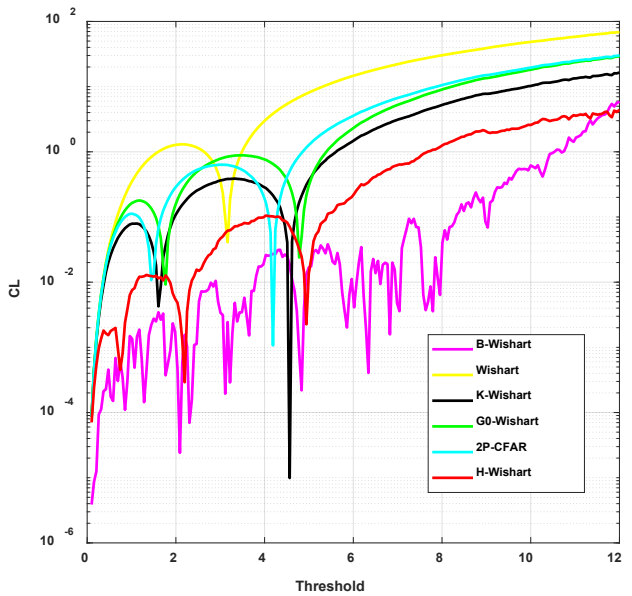

(a) $C_{L}$ in B-Wishart model

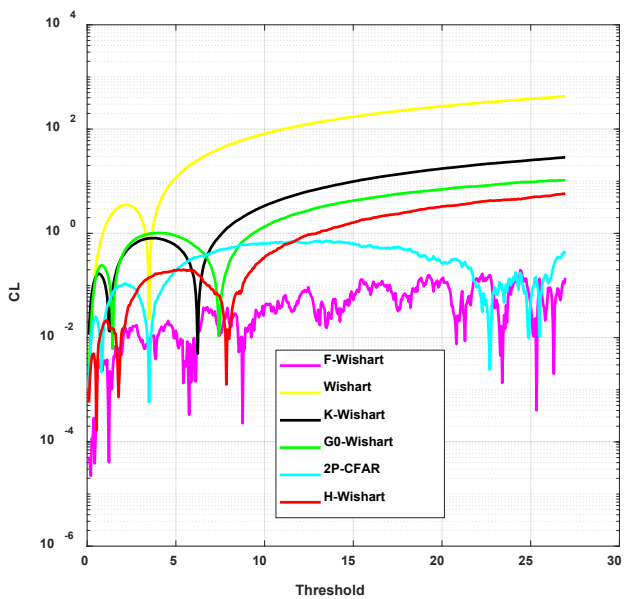

(b) $C_{L}$ simulating the data with a F-Wishart model

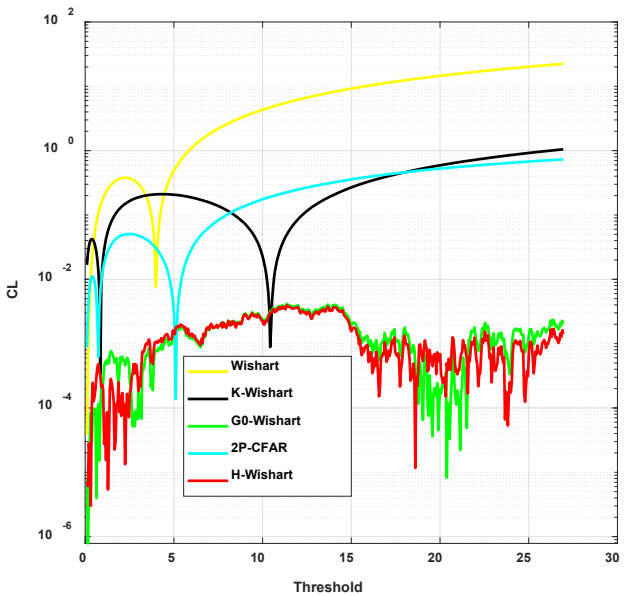

(c) $C_{L}$ simulating the data with a G0-Wishart model 


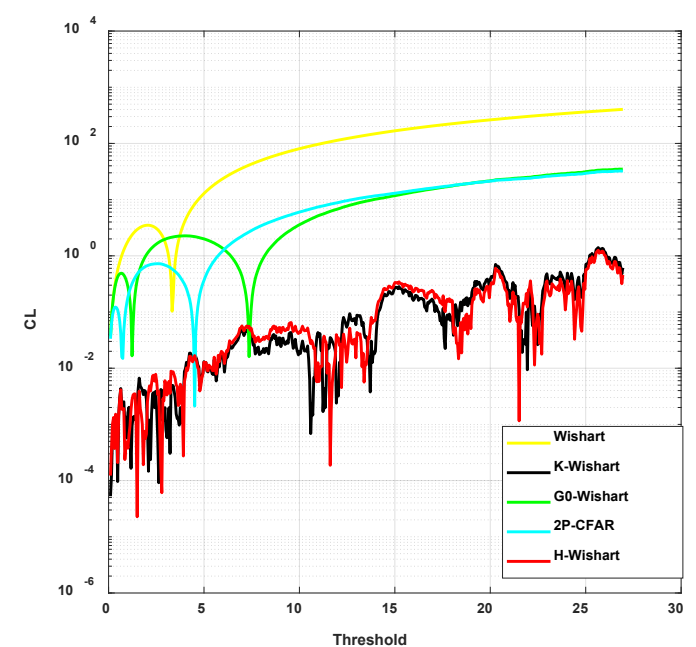

(d) $C_{L}$ simulating the data with a K-Wishart model

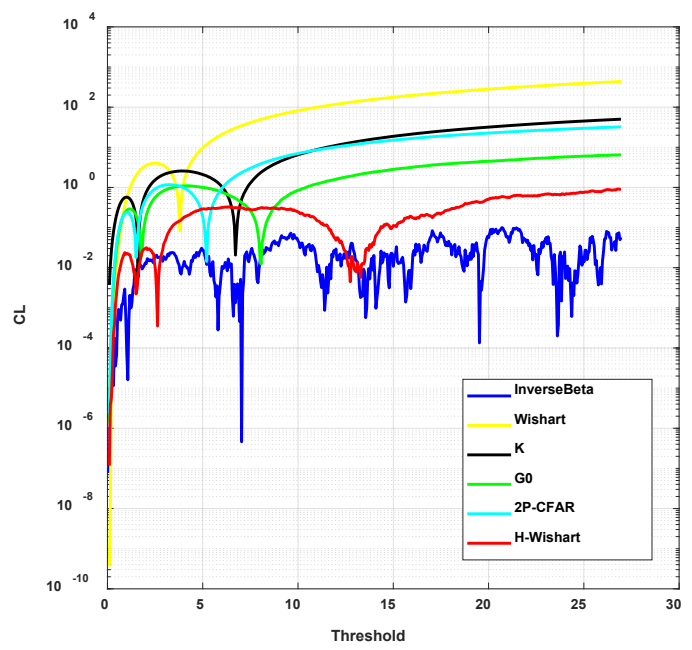

(e) $C_{L}$ simulating the data with a IB-Wishart model

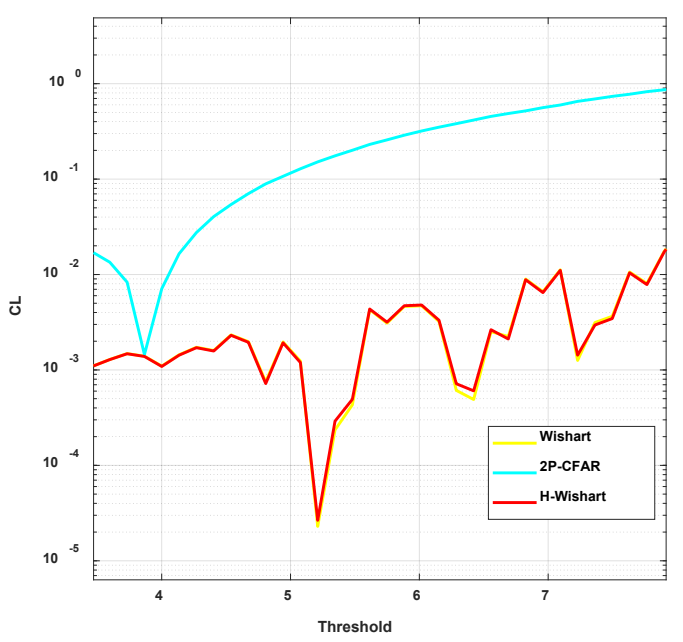

(f) $C_{L}$ simulating the data with a Wishart model

Fig. 7. The PFAs and $C_{L}$ in different Statistical models using different CFAR algorithms

From the PFAs and $C_{L}$ s in Fig 6 and Fig 7, it can be seen that the best performance in each CFAR maintenance is obtained by the corresponding model (i.e. the same model used for the simulations). This result is expected and verifies that the analytical derivation we made is correct and can be used. Though H-Wishart covers K-Wishart, G0-Wishart, F-Wishart, part of the B-Wishart and the IB-Wishart in $\mathrm{k} 3-\mathrm{k} 2$ plane, the CFAR performances are different. That is to say the $\mathrm{k} 3-\mathrm{k} 2$ logcumulants are not related with a unique statistical model and their CFAR performances may be different.

\section{Real data validation: testing on smaller areas}

The detection processing flow chart for real data is shown in Fig 8. Six subimages comprising sea areas mixed with ships were extracted from the original scene. The section were selected to include some variety on the set of log-cumulates considered. They represent at different parts of the log-cumulants space. The model recognition and goodness fit of the sub-images can be implemented by the log-cumulants method [34]. The subimages and the diagram of the $k 2-k 3$ plane are shown in Fig 9.

The purpose of this section is to assess the correctness of our derivations in real data. We need therefore to extract the statistics of the sea clutter, trying to minimize the effect of pixels which do not represent the sea. Therefore pixels from ships in sub-images should be removed. Considering we know the location of ships from visual inspection, we can simply remove their pixels from the image. As mentioned, the purpose of this session is to compare the different model and therefore we are producing an ideal dataset where the estimation of the clutter parameters is not affected by ships. In real operational scenario this is not possible and more complex procedures should be used.

In some studies, it is shown that the whole pixels in the sub-image can be used to estimate model parameters, because the number of ship pixels is much smaller than that of ocean pixels and hence the influence of ship pixels on the statistical properties of sea clutter is negligible [7]. In other studies, the brightest pixels can be removed [21][37] by suitable CFAR detectors, such as the order statistic CFAR (OS-CFAR), the trimmed mean CFAR (TM CFAR), the censored mean-level detector (CMLD) and the truncated statistic CFAR (TS-CFAR), etc [21]. Additionally outlier removal is usually done by data ranking or censoring with different restrictions [21]. Estimating the model parameters can be seen as a processing step and it is complementary to the following CFAR steps (carried out after the parameters are estimated). In this work, we only concentrate on the validity of the models and leave as a future work the stage were we remove the contamination from ships.

The processing steps are listed in the following:

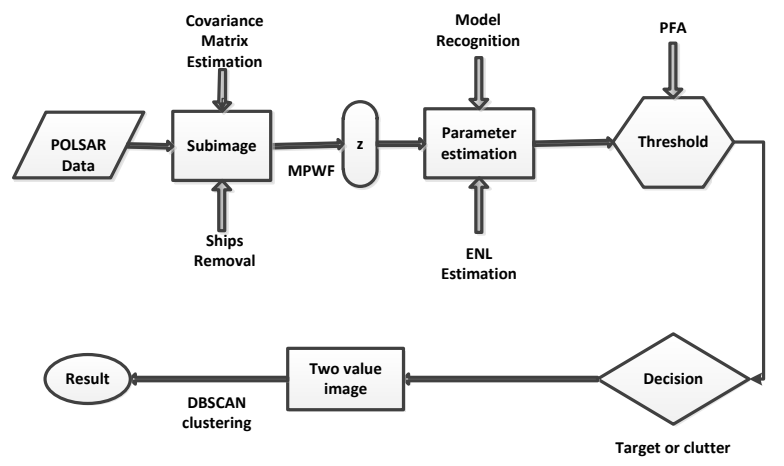

Fig. 8. The processing flow chart of CFAR algorithm 
Step1: we estimate the covariance matrix for the subimages. To avoid contamination in our parameter estimation we need to remove ships. In this work, a rectangle is applied to remove the obvious ship targets. Future work will be carried out in finding more appropriate operational solutions.

Step2. The MPWF processing for the PolSAR data is executed and the output $z$ is derived for each subimage.

Step3. In each subimage, the statistical model of clutter is selected based on the goodness of fit method [34] in the product model. By aid of the novel estimators in section IV, the distribution parameters are estimated. The ENL is estimated via the novel method proposed in [38], which is 3.4 here.

Step4. The analytical or bisection method is used to solve the complicated PFA equation to obtain the threshold $T$ according to the CFAR, which is set to be $10^{-3}$ here. When the threshold is obtained, it can be determined whether the pixel is a ship or clutter as follows:

$$
z=\operatorname{tr}\left(\boldsymbol{\Sigma}_{c}^{-1} \mathbf{C}\right) \underset{\text { Clutter }}{\stackrel{\text { Target }}{\gtrless}} T
$$

where $\boldsymbol{\Sigma}_{c}$ is the expectation of the clutter's covariance matrix and $\mathbf{C}$ is the multi-look covariance matrix. If the value is higher than the threshold, it is a ship; otherwise it is clutter, and then the binary image can be obtained.

Step5. The density-based clustering method named Density-Based Spatial Clustering of Applications with Noise [39] (DBSCAN) method is used to delete the solo points, and the final images are derived. This applies a second layer of false alarm rejections. It should be noted that we do not use clustering filter to evaluate the FAR and only apply those at the end.

Step6. The figure of merit (FOM) is used to evaluate the performance of the new CFAR methods after an MPWF, which is defined as [36][40]

$$
F O M=\frac{N_{t d}}{\left(N_{f a}+N_{g t}\right)}
$$

where $N_{t d}$ is the number of ships that are detected, $N_{f a}$ is the number of ships that are false alarms, $N_{g t}$ is the number of ships in the dataset, and FoM is the figure of merit (FOM). It should be noted that in the following figures a red circle means an omitted target, a yellow circle means a false target, and a yellow rectangle means one ship in the subimages.

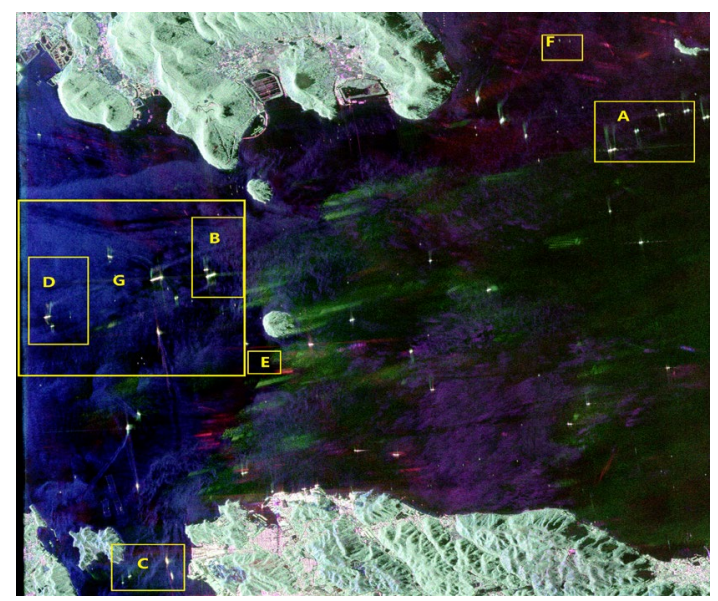

(a) Selected area

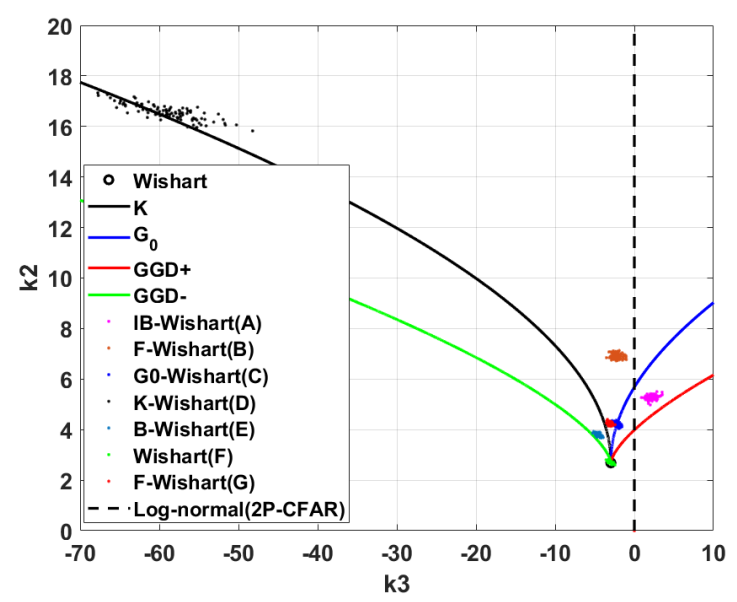

(b) Diagram of the $k 2-k 3$ plane

Fig. 9. The subimages and diagram of the log-cumulants in the $k 2-k 3$ plane of the AIRSAR data after ships are removed, and the Wishart is at origin

In Fig 10, there is only one true ship in the subimage E. The detection results of different CFAR thresholds are shown in the B-Wishart case. The size of the selected area is $134 \mathrm{x} 134$. In Table III, the key parameters to access the performance are shown. There are many false alarms in Wishart model. It can be seen that the B-Wishart detector gives the best performance in this case considering both the false alarm rate and FOM.

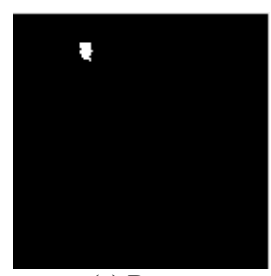

(a) Beta

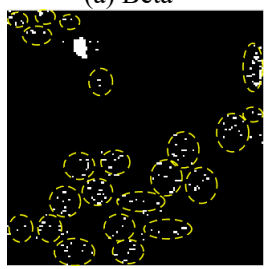

(d) Wishart

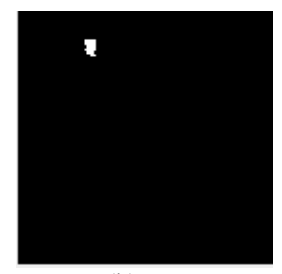

(b) $\mathrm{K}$

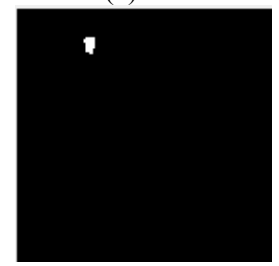

(e) 2P-CFAR

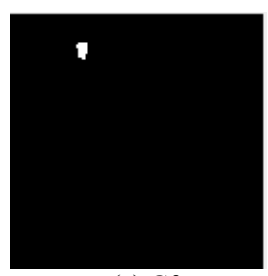

(c) G0

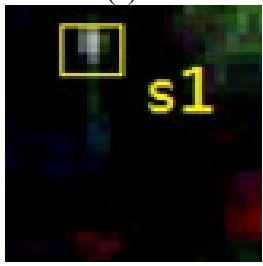

(f) Selected Area
Fig. 10. Results of different detectors in the Beta case

TABLE III

PERFORMANCES OF ALL DETECTORS IN THE BETA CASE

\begin{tabular}{cccccc}
\hline \hline Area & Method & $\hat{P}_{f a}$ & $N_{t d}$ & $N_{f a}$ & FoM(\%) \\
\hline \multirow{4}{*}{$\mathrm{E}$} & Beta & 0.0008 & 1 & 0 & 100 \\
\cline { 2 - 6 } & $\mathrm{K}$ & 0.00011 & 1 & 0 & 100 \\
\cline { 2 - 6 } & $\mathrm{G} 0$ & 0 & 1 & 0 & 100 \\
\cline { 2 - 6 } & Wishart & 0.01 & 1 & 22 & 4.35 \\
\cline { 2 - 6 } & 2PCFAR & 0 & 1 & 0 & 100 \\
\hline
\end{tabular}

In Fig 11, there are 3 true ships in the subimage $B$. The detection results of different CFAR methods are shown in the F-Wishart case. The size of the selected area is $325 \times 205$. In Table IV, the key parameters of the performance in different CFAR methods are shown." " means we did not calculate, because there are too many false alarms in the corresponding image, which will cause the FoM to be close to zero. The F-Wishart detector gives the best performance here. 


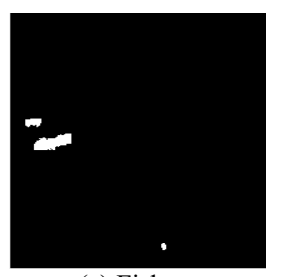

(a) Fisher

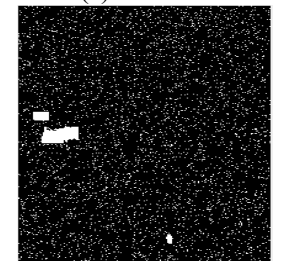

(d) Wishart

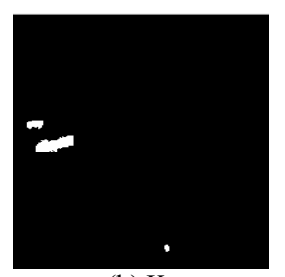

(b) $\mathrm{K}$

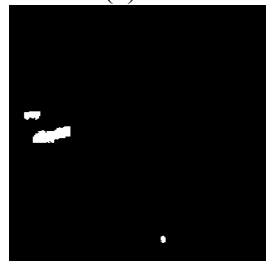

(e) 2P-CFAR

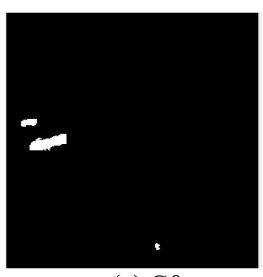

(c) G0

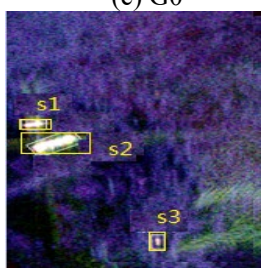

(f) Selected Area

Fisher case

TABLE IV

PERFORMANCES OF ALL DETECTORS IN FISHER CASE

\begin{tabular}{cccccc}
\hline \hline Area & Method & $\hat{P}_{f a}$ & $N_{t d}$ & $N_{f a}$ & FoM(\%) \\
\hline \multirow{4}{*}{ B } & Fisher & 0.001 & 3 & 0 & 100 \\
\cline { 2 - 6 } & $\mathrm{K}$ & 0.0013 & 3 & 0 & 100 \\
\cline { 2 - 6 } & $\mathrm{G} 0$ & 0.00036 & 3 & 0 & 100 \\
\cline { 2 - 6 } & Wishart & 0.654 & 3 & $/$ & $/$ \\
\cline { 2 - 6 } & 2PCFAR & 0.0011 & 3 & 0 & 100 \\
\hline
\end{tabular}

In Fig 12, there are 5 true ships in the subimage A. The detection results of different CFAR methods are shown in the IB-Wishart case. The size of the selected area is $305 \times 210$. There are many false alarms in other product models. In Table $\mathrm{V}$, the detection results are shown in the IB-Wishart case. The IB-Wishart detector gives the best performance in IB-Wishart distribution.

In Fig 13, there are 3 true ships in the selected area D. The detection results of different CFAR methods are shown in the $\mathrm{K}$-Wishart case. The size of the selected area is $153 \times 476$. It can be seen that the K-Wishart detector gives the best performance in K-Wishart distribution.

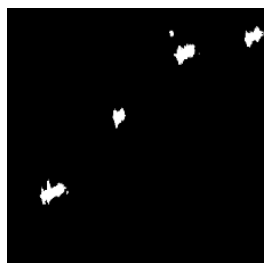

(a) IBeta

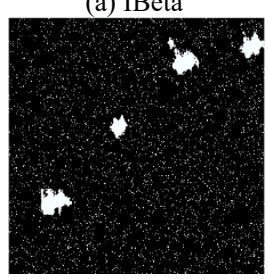

(d) Wishart

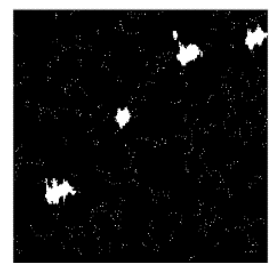

(b) $\mathrm{K}$

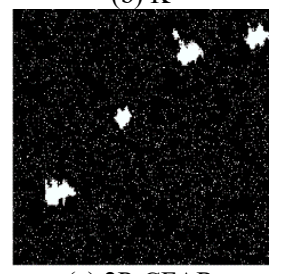

(e) 2P-CFAR

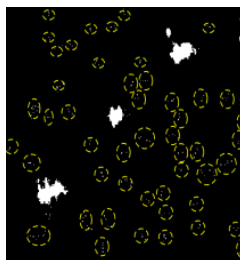

(c) G0

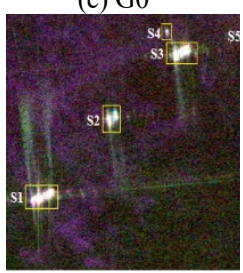

(f) Selected area
Fig. 12. Results of different detectors in the Ibeta case
TABLE V

PERFORMANCES OF ALL DETECTORS IN IBETA CASE

\begin{tabular}{cccccc}
\hline \hline \multirow{2}{*}{ Area } & Method & $\hat{P}_{f a}$ & $N_{t d}$ & $N_{f a}$ & FoM $(\%)$ \\
\hline \multirow{3}{*}{$\mathrm{A}$} & IBeta & 0.00099 & 5 & 0 & 100 \\
\cline { 2 - 6 } & $\mathrm{K}$ & 0.025 & 5 & $/$ & $/$ \\
\cline { 2 - 6 } & $\mathrm{G} 0$ & 0.008 & 5 & $/$ & $/$ \\
\cline { 2 - 6 } & Wishart & 0.632 & 5 & $/$ & $/$ \\
\cline { 2 - 6 } & 2PCFAR & 0.395 & 5 & $/$ & $/$ \\
\hline
\end{tabular}

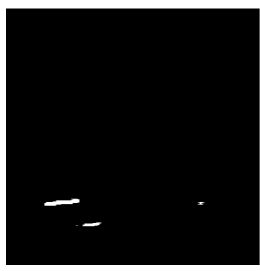

(a) $\mathrm{K}$

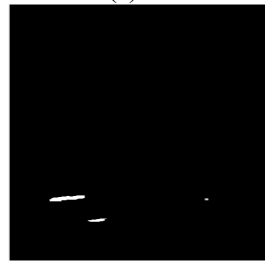

(d) 2P-CFAR

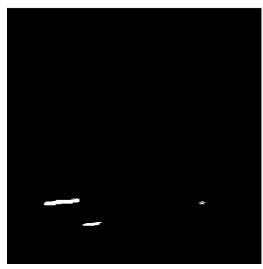

(b) G0

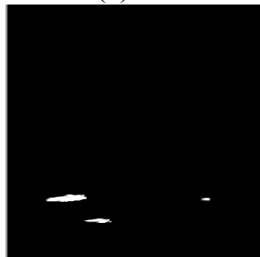

(e) H-Wishart

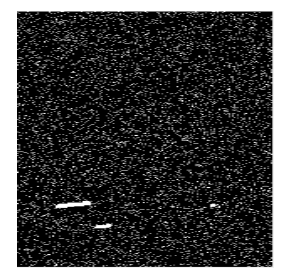

(c) Wishart

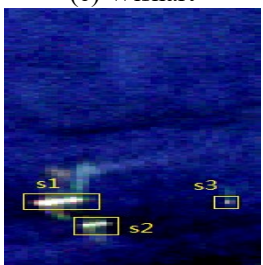

(f) Selected area
Fig. 13. Results of different detectors in the $\mathrm{K}$ case

In Table VI, the detection results of different CFAR methods are shown in the $\mathrm{K}-\mathrm{Wishart}$ case.

TABLE VI

PERFORMANCES OF ALL DETECTORS IN K CASE

\begin{tabular}{cccccc}
\hline \hline \multirow{2}{*}{ Area } & Method & $\hat{P}_{f a}$ & $N_{t d}$ & $N_{f a}$ & FoM $(\%)$ \\
\hline & $\mathrm{K}$ & 0.001 & 3 & 0 & 100 \\
\cline { 2 - 6 } $\mathrm{D}$ & $\mathrm{H}$ & 0.0011 & 3 & 0 & 100 \\
\cline { 2 - 6 } & Wishart & 0.641 & 3 & $/$ & $/$ \\
\cline { 2 - 6 } & 2PCFAR & 0 & 3 & 0 & 100 \\
\cline { 2 - 6 } & G0 & 0.00019 & 3 & 0 & 100 \\
\hline
\end{tabular}

In Fig 14 there are 4 true ships in the selected area $C$. The detection results of different CFAR methods are shown in the G0-Wishart case. The size of the selected area is $362 \times 324$. In Table VII the detection results of different CFAR methods have been shown in the K-Wishart case. There are 3 false alarms in $\mathrm{K}-\mathrm{Wishart}$ model. It can be seen that the G0-Wishart detector gives the best performance in the G0-Wishart distribution.

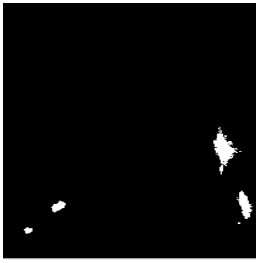

(a) G0

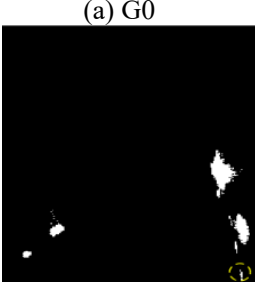

(d) 2P-CFAR

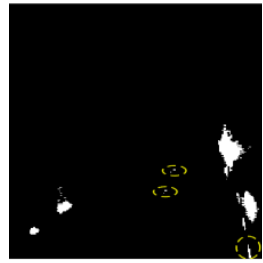

(b) $\mathrm{K}$

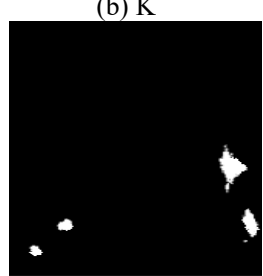

(e) H-Wishart

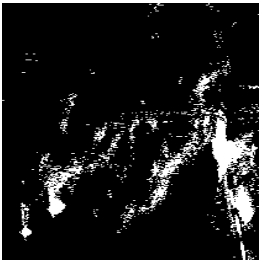

(c) Wishart

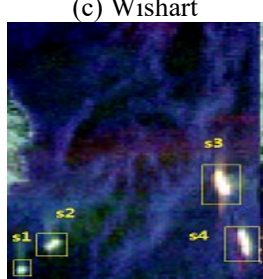

(f) Selected area
Fig. 14. Results of different detectors in the G0 case 
TABLE VII

PERFORMANCES OF ALL DETECTORS IN G0 CASE

\begin{tabular}{cccccc}
\hline \hline Area & Method & $\hat{P}_{f a}$ & $N_{t d}$ & $N_{f a}$ & $F o M(\%)$ \\
\hline \multirow{4}{*}{$\mathrm{C}$} & $\mathrm{G} 0$ & 0.00098 & 4 & 0 & 100 \\
\cline { 2 - 6 } & $\mathrm{H}$ & 0.001 & 4 & 0 & 100 \\
\cline { 2 - 6 } & $\mathrm{K}$ & 0.00143 & 4 & 3 & 57.14 \\
\cline { 2 - 6 } & Wishart & 0.058 & 4 & $/$ & $/$ \\
\cline { 2 - 6 } & 2PCFAR & 0.0013 & 4 & 1 & 80 \\
\hline
\end{tabular}

In Fig 15 there are 2 true ships in the selected area F. The detection results of different CFAR methods are shown in the Wishart case. The size of the selected area is $134 \times 134$. In Table VIII, the detection results of different CFAR models are shown in the Wishart case. It can be seen that the Wishart detector gives the best performance in Wishart distribution.

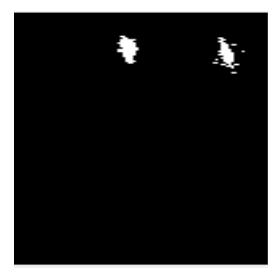

(a) Wishart

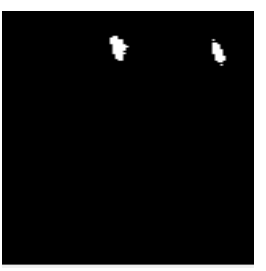

(b) 2P-CFAR

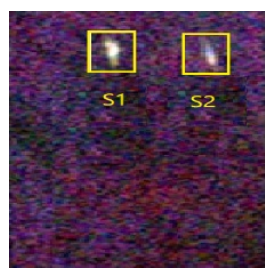

(c) Selected area
Fig. 15. Results of different detectors in the Wishart case

TABLE VIII

PERFORMANCES OF ALL DETECTORS IN WISHART CASE

\begin{tabular}{cccccc}
\hline \hline Area & Method & $\hat{P}_{f a}$ & $N_{t d}$ & $N_{f a}$ & FoM(\%) \\
\hline \multirow{2}{*}{ F } & Wishart & 0.001 & 2 & 0 & 100 \\
\cline { 2 - 6 } & 2PCFAR & 0.0001 & 2 & 0 & 100
\end{tabular}

\section{E. Real data validation: larger area}

The above experiments deal with few targets in the different sea clutters. In an operational use, the area assessed may be larger. Also, we decided here to test one simple technique to remove contamination from ships, although we are committed in working better techniques in the future. Therefore, it is more interesting to discuss all the detectors in scenes with a large quantity of targets. The wide area $\mathrm{G}$ is drawn from [36], and there are 22 targets in this scene as is shown in [36]. Here we modify the value of the constant false alarm to $1 \mathrm{e}-4$; I order to obtain results that are comparable with [36]. When priori information is not available, to estimate the PDF parameters we still need to remove ships. To do so, we set a threshold on the image removing the very bright pixels. Clearly, some smaller ship may be included, but since the area is very large it is likely their effect will be averaged out and they will not impact greatly the parameters estimation.

In the results the Wishart, $\mathrm{K}-\mathrm{W}$ ishart and $2 \mathrm{P}-\mathrm{CFAR}$ give many false alarms, while the F-Wishart and H-Wishart detectors give the best performance since the measured data may obey F-Wishart or H-Wishart distribution in the subimage G. The G0-Wishart gives a higher threshold which leads it to miss a true target. It can be found from the performance table that most of the detectors give a lower false alarm except the $\mathrm{K}-\mathrm{Wishart}$ detector. All the detectors work well in the low PFA case. The biases in the PFA may come from the goodness of fit between the measured data and theoretical models.

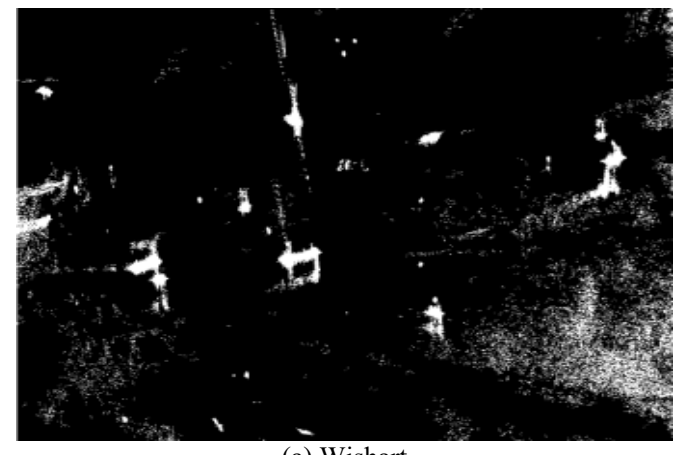

(a) Wishart

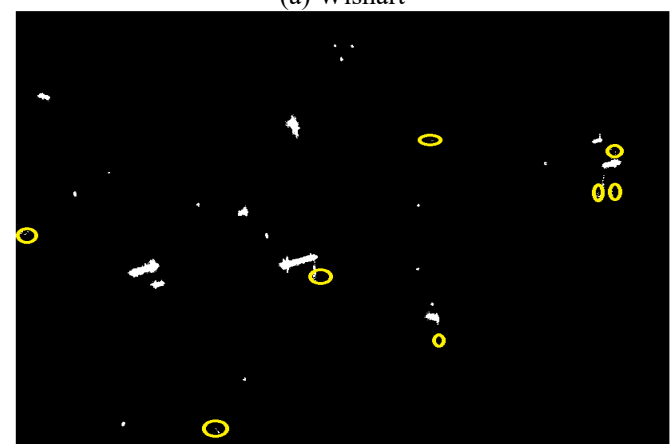

(b) 2P-CFAR

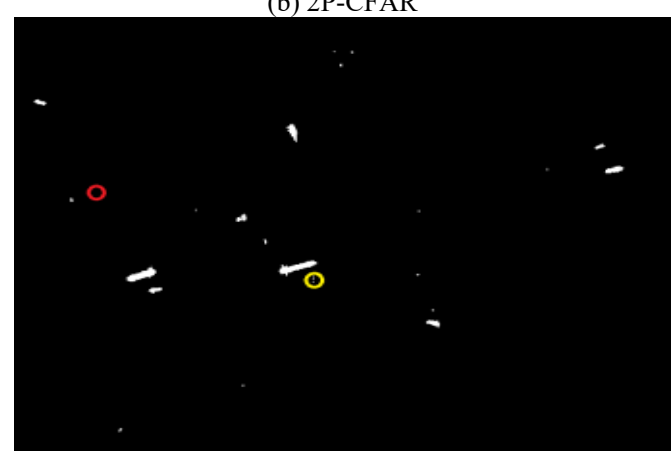

(c) G0

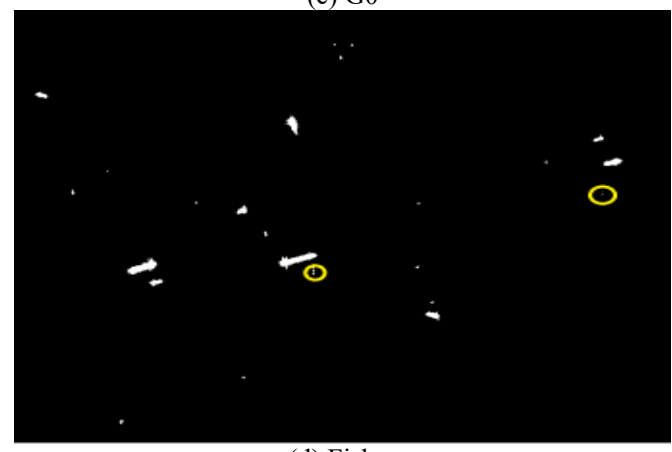

(d) Fisher

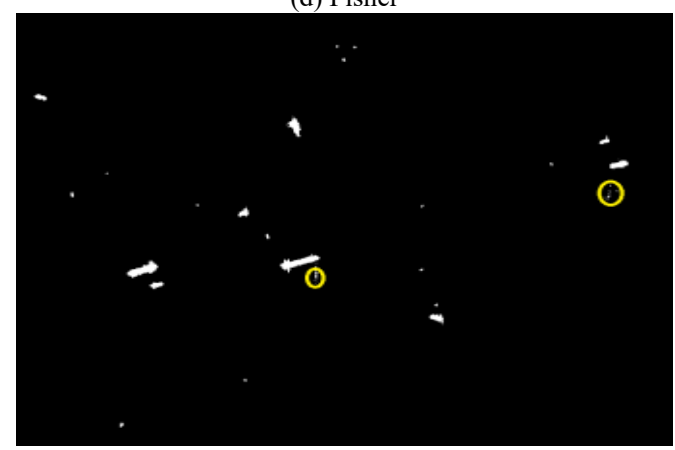

(e) $\mathrm{H}$ 


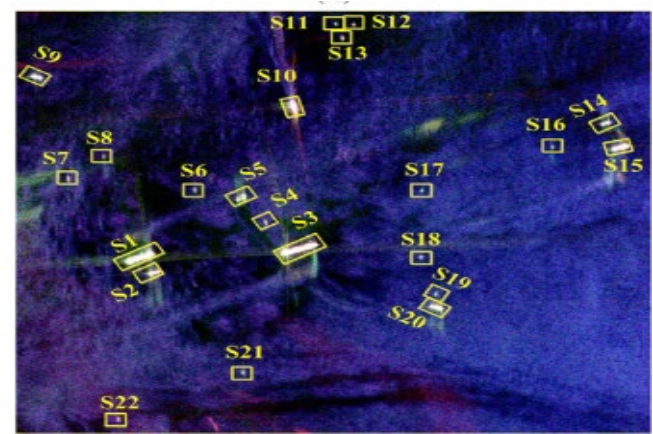

(f) Selected area[36]

Fig. 16 Results of different detectors in the many targets case

TABLE IX

PERFORMANCES OF ALL DETECTORS IN THIS CASE

\begin{tabular}{cccccc}
\hline \hline Area & Method & $\hat{P}_{f a}$ & $N_{t d}$ & $N_{f a}$ & $F o M(\%)$ \\
\hline \multirow{4}{*}{$\mathrm{G}$} & Fisher & $8.0 \mathrm{e}-06$ & 22 & 2 & 91.67 \\
\cline { 2 - 6 } & $\mathrm{K}$ & $1.8 \mathrm{e}-04$ & 22 & 23 & 48.89 \\
\cline { 2 - 6 } & $\mathrm{G} 0$ & $3.2 \mathrm{e}-06$ & 21 & 1 & 91.30 \\
\cline { 2 - 6 } & Wishart & $/$ & $/$ & $/$ & $/$ \\
\cline { 2 - 6 } & 2PCFAR & $1.4 \mathrm{e}-05$ & 22 & 8 & 73.33 \\
\cline { 2 - 6 } & $\mathrm{H}$ & $7.9 \mathrm{e}-06$ & 22 & 2 & 91.67 \\
\hline
\end{tabular}

\section{F. Computational Complexity}

Although the computing capability has been improved rapidly in recent years, the calculations in CFAR detection may still be heavy for a large PolSAR data. Here we want to discuss the computational burden of each algorithm. All algorithms are implemented in Matlab code. The performance measure is central processing unit (CPU) time, as measured by the Matlab function "tic" and "toc", on an Intel(R) Core(TM) i5-2400 CPU (a) 3.10GHz. Fig.17 displays average CPU time required per estimate calculation of CFAR threshold as a function of sample size. The parameter estimation method is based on MPWF in section IV via Newton's method, and the solution of the threshold is based on the analytical expressions of PFA in section III via bisection method (analytical methods are used in both Wishart case and 2P-CFAR case).

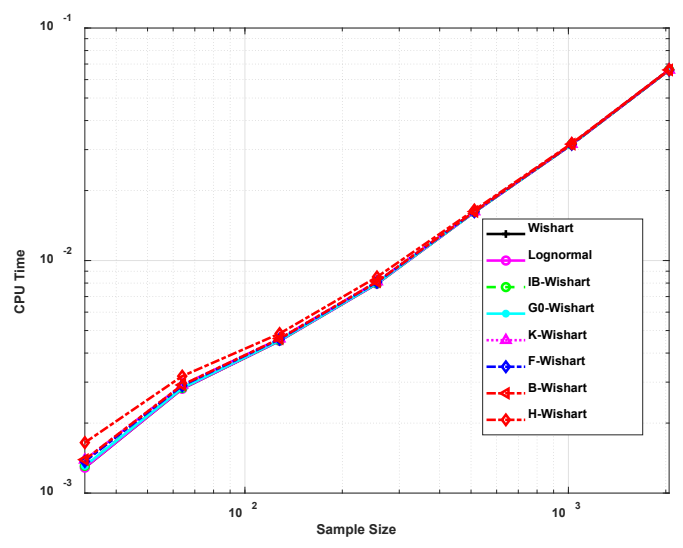

(a) Parameter estimation time

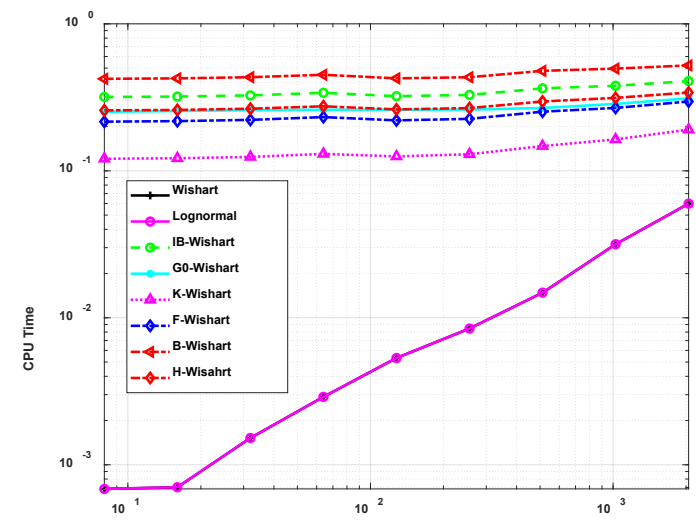

(b) Total time including threshold solution Fig. 17. Computational complexity

Fig 17(a) shows the parameter estimation time for different models. All estimators in different models have almost the same estimation time as $1 \mathrm{e}-3$ second per sample. Clearly, this increases with the sample numbers projecting this time we can expect around 1s for each Megapixel. Fig 17(b) shows the total time including both parameter estimation time and threshold solution time. It can be seen the CPU time in Wishart and lognormal case is the shortest, which increases with the sample number. This is because the total time is only impacted by the time for estimated parameters. The most of the algorithms are flat with increasing the sample size. This is because the total time is almost determined by the time of threshold solution.

For this purpose, since the Wishart and 2P-CFAR methods have the analytical threshold, both are suitable for fast computation. It also shows that $2 \mathrm{P}-\mathrm{CFAR}$ misses ships, while Wishart CFAR generates large false alarms which may be deleted by severe clustering algorithms. In Fig 18, the simulation results of Wishart detector are presented when clustering parameters are changed. Here $\varepsilon=10$, and MinPts $=8$, which is suitable for large ships, while may cause missed alarms for small ships. The simulated data is the same as that in part B including Beta, K, G0, IBeta and Fisher distributed clutter. The number of simulated ships is also 15 , which are also Wishart distributed.

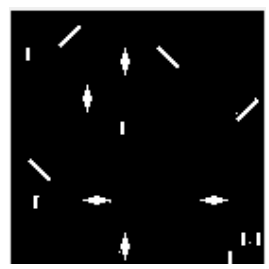

(a) Beta

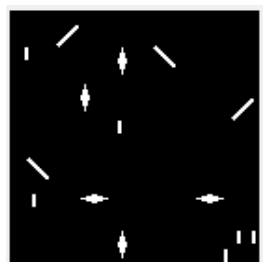

(d) IBeta

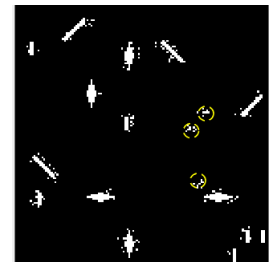

(b) $\mathrm{K}$

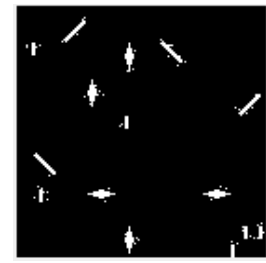

(e) Fisher

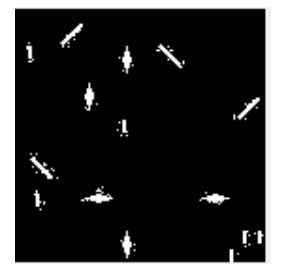

(c) G0

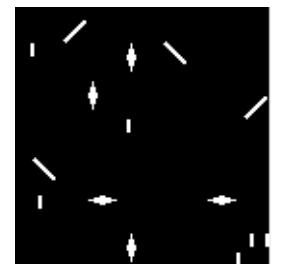

(f) Sim-ships
Fig. 18. Comparisons of Wishart detectors in the different clutter models 
TABLE X

PERFORMANCES OF WISHART DETECTOR IN THE ALL MODELS

\begin{tabular}{ccccc}
\hline \hline Area & $\hat{P}_{f a}$ & $N_{t d}$ & $N_{f a}$ & $F o M(\%)$ \\
\hline $\mathrm{E}($ Beta $)$ & 0.012 & 15 & 0 & 100 \\
\hline $\mathrm{D}(\mathrm{K})$ & 0.619 & 15 & 3 & 83.33 \\
\hline $\mathrm{C}(\mathrm{G} 0)$ & 0.598 & 15 & 0 & 100 \\
\hline $\mathrm{A}($ IBeta $)$ & 0.265 & 15 & 0 & 100 \\
\hline $\mathrm{B}$ (Fisher) & 0.498 & 15 & 0 & 100 \\
\hline
\end{tabular}

The performances of Wishart detector in all environments have been presented in Table X. It can be seen that when the clustering algorithm becomes severe, the Wishart detector may get very good performances. In fact the clustering parameters are hard to determine and it may miss many small ships. It is still a big problem how to determine the clustering parameters and make the small ships detectable.

\section{CONCLUSION}

In this paper, analytical CFAR algorithms have been derived for ship detection in MPWF Pol-SAR images in multivariate product models. The analytical expressions are derived for the PDF and PFA in different distributed clutters including Wishart, K-Wishart, G0-Wishart, F-Wishart, B-Wishart, IB-Wishart and H-Wishart in PolSAR imagery. The novel parameter estimators were also proposed for all multivariate product models based on MPWF and log-cumulants. With the increase of the degree of nonhomogeneous, the performance of $k 2 k 3$-MPWF estimator is better than that of the $k 1 k 2$-MPWF. The $k 2 k 3$ estimator may give the best estimation in $\mathrm{H}-\mathrm{Wishart}$ case, while it need more sample numbers. The threshold problem is solved by both analytical forms in Wishart and 2P-CFAR cases and the numerical method of bisection in other product models. It can be concluded that the performance of ship detection in complicated clutter is decided by the accuracy of the statistical models. The closer the clutter's distribution is to the statistical model, the better the performance of ship detection will be. For fast CFAR calculations, the Wishart method is proposed. Its performance is better than that of the commonly used 2P-CFAR method, especially in a low target-clutter-rate (TCR) case.

In fact the phenomenon that ships will stay in the clutter window will influence the statistics of CFAR strongly in multi targets situation. The influence of multi targets on the clutter statistics must be considered and can be reduced by the truncated statistic CFAR (TS-CFAR). But until now the TS-CFAR is only used in the single polarization SAR images. How to extend it to the full polarimetric SAR still needs to be studied.

\section{APPENDIX A}

According to literature [27], $z$ obeys a Gamma distribution in the Wishart case:

$$
z=\frac{1}{L} \sum_{i=1}^{L} y_{i}^{H} \Sigma^{-1} y_{i} \sim \gamma\left(L d, \frac{1}{L}\right)
$$

where $\gamma(\alpha, \beta)$ denotes a Gamma distribution with shape parameter $\alpha$, and scale parameter $\beta . \gamma(\alpha, \beta)$ can be represented as

$$
\gamma(\alpha, \beta)=\frac{1}{\Gamma(\alpha)} \frac{1}{\beta}\left(\frac{x}{\beta}\right)^{\alpha-1} e^{-\frac{x}{\beta}}
$$

Then, the probability density function of $z$ is

$$
p_{z}\left(L d, \frac{1}{L}\right)=\frac{L^{L d} z^{L d-1}}{\Gamma(L d)} e^{-L z}
$$

If the detection threshold is assumed to be $T$, the false alarm rate can be determined as

$$
P_{f a}=\int_{T}^{\infty} p_{z}\left(L d, \frac{1}{L}\right) d z=\frac{\Gamma[d L, L T]}{\Gamma[d L]}
$$

$P_{f a}$ is the false alarm rate, $d$ is the dimension of the scattering vector, $L$ is the number of looks. The gamma function and the incomplete gamma function are defined as follows[28]

$$
\Gamma(x)=\int_{0}^{\infty} e^{-t} t^{x-1} d t, \Gamma(a, x)=\int_{x}^{\infty} e^{-t} t^{a-1} d t
$$

\section{APPENDIX B}

In K-Wishart case, it can be determined that $x$ obeys a Gamma distribution [27]

$$
x \sim \gamma\left(L d, \frac{1}{L}\right)=\frac{L^{L d} x^{L d-1}}{\Gamma(L d)} e^{-L x}
$$

$\tilde{\tau}$ obeys the unitary Gamma distribution

$$
\tilde{\tau} \sim \gamma\left(\alpha, \frac{1}{\alpha}\right)=\frac{\alpha^{\alpha}}{\Gamma(\alpha)} \tilde{\tau}^{\alpha-1} e^{-\alpha \tilde{\tau}}
$$

where $\alpha$ is the shape parameter. Therefore, $z$ will obey the Gamma-Gamma distribution, which is [29]

$$
f_{z}(\mathrm{z} ; \alpha, L, d)=\frac{2(\alpha L)^{\frac{\alpha+L d}{2}} z^{\frac{\alpha+L d}{2}-1}}{\Gamma(L d) \Gamma(\alpha)} K_{\alpha-L d}\left[2(\alpha L z)^{1 / 2}\right]
$$

where $K_{\alpha-L d}(\cdot)$ is the second type of modified Bessel function, which is defined as [29]

$$
K_{v}(z)=\frac{\Gamma(v+1 / 2)(2 z)^{v}}{\sqrt{\pi}} \int_{0}^{\infty} \frac{\cos u}{\left(u^{2}+z^{2}\right)^{v+1 / 2}} d u
$$

Via the characteristics of the MeijerG-function [30]

$$
G_{0,2}^{2,0}[x \mid a, b]=2 x^{1 / 2(a+b)} K_{a-b}\left(2 x^{1 / 2}\right)
$$

and the integration equation [30][41] is

$$
\begin{aligned}
& \int_{0}^{y} x^{\alpha-1} G_{p, q}^{m, n}\left[\omega x \mid \begin{array}{l}
\left(a_{p}\right) \\
\left(b_{q}\right)
\end{array}\right] \\
& =y^{\alpha} G_{p+1, q+1}^{m, n+1}\left[\omega y \mid \begin{array}{l}
a_{1}, \ldots a_{n}, 1-\alpha, a_{n+1}, \ldots a_{p} \\
b_{1}, \ldots b_{m},-\alpha, b_{m+1}, \ldots b_{q}
\end{array}\right]
\end{aligned}
$$

When the threshold is $T$, we can obtain the cumulate density function (CDF) of $\mathrm{z}$

$$
\begin{aligned}
& F_{\mathrm{K}}(T ; \alpha, L, d)=\int_{0}^{T} f_{z}(\mathrm{z} ; \alpha, L, d) d z \\
& =\frac{1}{\Gamma(L d) \Gamma(\alpha)} G_{1,3}^{2,1}\left[\alpha L \mid \begin{array}{c}
1 \\
\alpha, L d, 0
\end{array}\right]
\end{aligned}
$$

The probability density function of the false alarm can be derived as follows 


$$
\begin{aligned}
& P_{f a}=1-F_{\mathrm{K}}(T ; \alpha, L, d) \\
& =1-\frac{1}{\Gamma(L d) \Gamma(\alpha)} G_{1,3}^{2,1}\left[\alpha L T \mid \begin{array}{c}
1 \\
\alpha, L d, 0
\end{array}\right]
\end{aligned}
$$

where $G(\bullet)$ is MeijerG function [30].

$$
\begin{aligned}
& G_{p, q}^{m, n}\left[z \mid \begin{array}{l}
a_{1}, \cdots, a_{p} \\
b_{1}, \cdots, b_{q}
\end{array}\right]=\frac{1}{2 \pi i} \\
& \times \int_{L} \frac{\prod_{j=1}^{m} \Gamma\left(b_{j}-s\right) \prod_{j=1}^{n} \Gamma\left(1-a_{j}+s\right)}{\prod_{j=m+1}^{q} \Gamma\left(1-b_{j}+s\right) \prod_{j=n+1}^{p} \Gamma\left(a_{j}-s\right)} z^{s} d s
\end{aligned}
$$

\section{APPENDIX C}

In G0-Wishart case, it means that $\tilde{\tau}$ obeys a unitary inverse Gamma distribution, which is denoted as[7]:

$$
f(\tilde{\tau} ; \lambda)=\frac{(\lambda-1)^{\lambda}}{\Gamma(\lambda)} \tilde{\tau}^{-\lambda-1} \exp \left(\frac{1-\lambda}{\tilde{\tau}}\right) \quad(\lambda, \tilde{\tau}>0)
$$

where $\lambda$ is the shape parameter, and $\tilde{\tau}$ is the unitary texture variable. Therefore, the probability density function (PDF) of the variable $z=\tilde{\tau} x$ after an MPWF is presented as

$$
f_{\mathrm{G} 0}(z ; \lambda, L, d)=\int_{0}^{+\infty} \frac{1}{\tilde{\tau}} f\left(x ; L d, \frac{1}{L}\right) f(\tilde{\tau} ; \lambda) d \tilde{\tau}
$$

Replacing $x=z / \tilde{\tau}$ in the above equation, we can obtain

$$
\begin{aligned}
& f_{\mathrm{G} 0}(z ; \lambda, L, d)=\frac{L^{L d} z^{L d-1}(\lambda-1)^{\lambda}}{\Gamma(L d) \Gamma(\lambda)} \\
& \times \int_{0}^{+\infty} \frac{1}{\tilde{\tau}^{L d+\lambda+1}} \exp \left(\frac{1-\lambda-L z}{\tilde{\tau}}\right) d \tilde{\tau}
\end{aligned}
$$

where $L$ is the number of looks, $d$ is the dimension of the polarimetric scattering vector, $z$ is the detection variable after a MPWF, and $\lambda$ is the shape parameter of the inverse Gamma distribution. Using the integration equations in [42]

$$
h^{-1} \alpha^{s / h} \Gamma(-s / h)=\int_{0}^{+\infty} x^{s-1} \exp \left(-\alpha x^{-h}\right) d x
$$

Eq. (C.3) can be simplified to

$$
f_{\mathrm{G} 0}(z ; \lambda, L, d)=\frac{L^{L d} z^{L d-1}(\lambda-1)^{\lambda}}{B(L d, \lambda)(\lambda+L z-1)^{L d+\lambda}}
$$

where $B(a, b)$ is the Beta function[28]

$$
B(a, b)=\frac{\Gamma(a) \Gamma(b)}{\Gamma(a+b)}
$$

If the threshold is $T$, the CDF of $z$ after an MPWF is

$$
F_{\mathrm{G} 0}(T ; \lambda, L, d)=\int_{0}^{T} \frac{L^{L d}(\lambda-1)^{\lambda}}{B(L d, \lambda)} \frac{z^{L d-1}}{(\lambda+L z-1)^{L d+\lambda}} d z
$$

Via the integration equation in [30]

$$
\int_{0}^{u} \frac{x^{\mu-1} d x}{(1+\beta x)^{v}}=\frac{u^{\mu}}{\mu}{ }_{2} F_{1}(v, \mu ; 1+\mu ;-\beta u)
$$

Eq.(C.6) can be simplified as

$$
\begin{aligned}
& F_{\mathrm{G} 0}(T ; \lambda, L, d)=\frac{L^{L d}}{B(L d, \lambda)(\lambda-1)^{L d}} \int_{0}^{T} \frac{z^{L d-1}}{\left(1+\frac{L}{\lambda-1} z\right)^{L d+\lambda}} d z \\
& =\frac{1}{B(L d, \lambda) L d}\left(\frac{L T}{\lambda-1}\right)_{2}^{L d} F_{1}\left(L d+\lambda, L d ; 1+L d ;-\frac{L T}{\lambda-1}\right)
\end{aligned}
$$

It is easy to obtain the probability density function of false alarm,

$$
\begin{aligned}
& P_{f a}=1-F_{\mathrm{G} 0}(T ; \lambda, L, d) \\
& =1-\frac{1}{B(L d, \lambda) L d}\left(\frac{L T}{\lambda-1}\right)^{L d} \\
& \times{ }_{2} F_{1}\left(L d+\lambda, L d ; 1+L d ;-\frac{L T}{\lambda-1}\right)
\end{aligned}
$$

where ${ }_{2} F_{1}(\cdot)$ is the Gaussian Hyper geometric function[28].

\section{APPENDIX D}

In F-Wishart case, the PDF of the unitary Fisher texture $\tilde{\tau}$ is presented as follows [23].

$$
\tilde{\tau} \sim \phi_{\mathrm{F}}(u, v)=\frac{1}{B(u, v)} \frac{\omega(\omega \tilde{\tau})^{v-1}}{(\omega \tilde{\tau}+1)^{u+v}}
$$

where $u$ and $v$ are the freedom of the Fisher distribution, $\Gamma(\cdot)$ is the Gamma function, and $\omega=v /(u-1)$.

The PDF of the output $z$ after a MPWF should be

$$
f_{\mathrm{F}}(z ; u, v, L, d)=\int_{0}^{+\infty} \gamma\left(L d, \frac{1}{L}\right) \phi_{\mathrm{F}}(u, v) J d \tilde{\tau}
$$

where $J$ is the Jacobian value, $J=\frac{\partial(x)}{\partial(z)}=\frac{1}{\tilde{\tau}} \tau=\omega \tilde{\tau}$, and $x=\frac{z}{\tilde{\tau}}$.

The following equation can then be

$$
\begin{aligned}
& f_{\mathrm{F}}(z ; u, v, L, d)=\frac{[L z \omega]^{L d}}{z \Gamma(L d)} \frac{1}{B(u, v)} \\
& \times \int_{0}^{+\infty} \tau^{v-L d-1}(1+\tau)^{-u-v} \exp \left(-L z \omega \tau^{-1}\right) d \tau
\end{aligned}
$$

where $\tau$ can be replaced by $m=1 / \tau$, and the following can be obtained

$$
\begin{aligned}
& f_{\mathrm{F}}(z ; u, v, L, d)=\frac{[L z \omega]^{L d}}{z \Gamma(L d)} \frac{1}{B(u, v)} \\
& \times \int_{0}^{+\infty} m^{u+L d-1}(1+m)^{-u-v} \exp (-L z \omega m) d m
\end{aligned}
$$

Using the definition of the KummerU function[28],

$$
U(a, b, z)=\frac{1}{\Gamma(a)} \int_{0}^{+\infty} \exp (-z t) t^{a-1}(1+t)^{b-a-1} d t
$$

Eq. (D.4) can then be simplified as follows

$$
\begin{aligned}
& f_{\mathrm{F}}(z ; u, v, L, d)=\frac{[L z \omega]^{L d}}{z \Gamma(L d)} \frac{1}{B(u, v)} \\
& \times \Gamma(u+L d) U[u+L d, L d+1-v, L z \omega]
\end{aligned}
$$

This is just the PDF of the variable $z$. 
If the threshold is $T$, the CDF can be calculated

$$
\begin{aligned}
& F_{\mathrm{F}}(T ; u, v, L, d)=\frac{[L \omega]^{L d}}{\Gamma(L d)} \frac{1}{B(u, v)} \\
& \times \int_{0}^{T} \int_{0}^{+\infty} m^{u+L d-1}(1+m)^{-u-v} \exp (-L z \omega m) z^{L d-1} d m d z
\end{aligned}
$$

where $m=1 / \tau$.Via the relationship between the incomplete Gamma function and general hyper geometric function[28]:

$$
\Gamma(a, x)=\int_{0}^{x} e^{-t} t^{a-1} d t=a^{-1} x^{a}{ }_{1} F_{1}(a ; a+1 ;-x)
$$

where $\Gamma(a, x)$ is the incomplete Gamma function and ${ }_{p} F_{q}\left(a_{1}, \cdots, a_{p} ; b_{1}, \cdots, b_{q} ; z\right)$ is the general hyper geometric function. We can then obtain

$$
\begin{aligned}
& F_{\mathrm{F}}(T ; u, v, L, d)=\frac{1}{B(u, v)} \frac{[L T \omega]^{L d}}{\Gamma(L d) L d} \\
& \times \int_{0}^{+\infty} m^{u+L d-1}(1+m)^{-u-v}{ }_{1} F_{1}[L d ; L d+1 ;-L T \omega m] d m
\end{aligned}
$$

Using the relationship between the Hyper geometric function and MeijerG-function [28], we have

$$
\begin{gathered}
{ }_{p} F_{q}\left(a_{1}, \cdots, a_{p} ; b_{1}, \cdots, b_{q} ;-x\right)=\frac{\prod_{j=1}^{q} \Gamma\left(b_{j}\right)}{\prod_{j=1}^{p} \Gamma\left(a_{j}\right)} \\
\times x G_{p, q+1}^{1, p}\left(x \mid \begin{array}{l}
-a_{1}, \cdots,-a_{p} \\
-1,-b_{1}, \cdots,-b_{q}
\end{array}\right) \quad p \leq q+1
\end{gathered}
$$

where $G_{p, q}^{m, n}\left(x \mid \begin{array}{l}a_{1}, \cdots, a_{p} \\ b_{1}, \cdots, b_{q}\end{array}\right)$ is the MeijerG-function. We can then obtain

$$
\begin{aligned}
& F_{\mathrm{F}}(T ; u, v, L, d)=\frac{[L T \omega]^{L d+1} \Gamma(L d+1)}{\Gamma^{2}(L d) B(u, v) L d} \\
& \times \int_{0}^{+\infty} m^{L d+u}(1+m)^{-u-v} G_{1,2}^{1,1}\left(L T m \omega \mid \begin{array}{l}
-L d \\
-1,-L d-1
\end{array}\right) d m
\end{aligned}
$$

Using the integration equation in the literature[30]:

$$
\begin{aligned}
& \int_{0}^{\infty} x^{\rho-1}(x+\beta)^{-\sigma} G_{p, q}^{m, n}\left(a x \mid \begin{array}{l}
a_{1}, \cdots, a_{p} \\
b_{1}, \cdots, b_{q}
\end{array}\right) d x \\
& =\frac{\beta^{\rho-\sigma}}{\Gamma(\sigma)} G_{p+1, q+1}^{m+1, n+1}\left(a \beta \mid \begin{array}{l}
1-\rho, a_{1}, \cdots, a_{p} \\
\sigma-\rho, b_{1}, \cdots, b_{q}
\end{array}\right)
\end{aligned}
$$

we obtain the CDF

$$
\begin{aligned}
& F_{\mathrm{F}}(T ; u, v, L, d)=\frac{[L T \omega]^{L d+1} \Gamma(L d+1)}{\Gamma^{2}(L d) \Gamma(u) \Gamma(v) L d} \\
& \times G_{2,3}^{2,2}\left(L T \omega \mid \begin{array}{l}
-L d-u,-L d \\
v-L d-1,-1,-L d-1
\end{array}\right)
\end{aligned}
$$

Therefore, the PDF of the false alarm is

$$
\begin{aligned}
& P_{f a}=1-\frac{[L T \omega]^{L d+1} \Gamma(L d+1)}{\Gamma^{2}(L d) \Gamma(u) \Gamma(v) L d} \\
& \times G_{2,3}^{2,2}\left(L T \omega \mid \begin{array}{l}
-L d-u,-L d \\
v-L d-1,-1,-L d-1
\end{array}\right)
\end{aligned}
$$

\section{APPENDIX E}

In IB-Wishart case, the unitary texture variable $\tilde{\tau}$ obeys the inverse Beta distribution [23]

$$
\begin{aligned}
& \tilde{\tau} \sim \phi_{\mathrm{IB}}(u, v)=\frac{1}{B(u, v)} \\
& \times \eta(\eta \tilde{\tau}-1)^{u-1}(\eta \tilde{\tau})^{-u-v}, \quad \tilde{\tau} \in\left[\frac{1}{\eta}, \infty\right]
\end{aligned}
$$

where $\eta=(u+v-1) /(v-1)$, and $u$ and $v$ are the freedom of the beta distribution. The variable $\tau=\eta \tilde{\tau}$ also obeys inverse beta distribution[23]:

$$
f_{\mathrm{IB}}(\tau)=\frac{1}{B(u, v)}(\tau-1)^{u-1} \tau^{-u-v}, \quad \tau \in[1, \infty]
$$

Therefore, the PDF of the output variable $z$ is

$$
f_{\mathrm{IB}}(z ; u, v, L, d)=\int_{0}^{+\infty} \gamma\left(L d, \frac{1}{L}\right) \phi_{\mathrm{IB}}(u, v) J d \tilde{\tau}
$$

where $J$ is the Jacobian value, $J=\frac{\partial(x)}{\partial(z)}=\frac{1}{\tilde{\tau}}$. Using $\tau=\eta \tilde{\tau}$ and $x=\frac{z}{\tilde{\tau}}$, we-obtain

$$
\begin{aligned}
& f_{\mathrm{IB}}(z ; u, v, L, d)=\frac{[L z \eta]^{L d}}{z \Gamma(L d)} \frac{1}{B(u, v)} \\
& \times \int_{1}^{\infty} \tau^{-v-L d-u}(\tau-1)^{u-1} \exp \left(-L z \eta \tau^{-1}\right) d \tau
\end{aligned}
$$

Replacing the variable $m$ with $m=1 / \tau$, we-obtain

$$
\begin{aligned}
& f_{\mathrm{IB}}(z ; u, v, L, d)=\frac{[L z \eta]^{L d}}{z \Gamma(L d)} \frac{1}{B(u, v)} \\
& \times \int_{0}^{1} m^{-1+v+L d}(1-m)^{u-1} \exp (-L z \eta m) d m
\end{aligned}
$$

Using the definition of the KummerM function [28]

$$
M(a, b, z) \Gamma(a) \frac{\Gamma(b-a)}{\Gamma(b)}=\int_{0}^{1} \exp (z t)(1-t)^{b-a-1} \mathrm{t}^{a-1} d t
$$

we have

$$
\begin{aligned}
& f_{\mathrm{IB}}(z ; u, v, L, d)=\frac{[L z \eta]^{L d}}{z B(L d, v)} \frac{\Gamma(u+v)}{\Gamma(L d+v+u)} \times \\
& M(L d+v, L d+v+u,-L z \eta)
\end{aligned}
$$

Using the definition of the WhittakerM function

$$
\mathrm{M}_{k, \mu}(z)=z^{\mu+\frac{1}{2}} \exp \left(-\frac{z}{2}\right) \mathrm{M}\left(\mu-k+\frac{1}{2}, 2 \mu+1, z\right)
$$

Substituting $\quad k=\frac{u-L d-v}{2} \quad, \quad \mu=\frac{L d+v+u-1}{2} \quad$ and $z=-L z \eta$ into Eq.(E.6), then the equation can be expressed in another form 


$$
\begin{aligned}
& f_{\mathrm{IB}}(z ; u, v, L, d)=\frac{[-L \eta z]^{-\frac{L d+v+u}{2}}[L \omega z]^{L d}}{z B(L d, v)} \frac{\Gamma(u+v)}{\Gamma(L d+v+u)} \\
& \times \exp \left(\frac{-L z \eta}{2}\right) \mathrm{M}_{\frac{u-L d-v}{2}, \frac{L d+v+u-1}{2}}(-L z \eta)
\end{aligned}
$$

If the threshold is $T$, the CDF should be

$$
\begin{aligned}
& F_{\mathrm{IB}}(T ; u, v, L, d)=\frac{[L \eta]^{L d}}{\Gamma(L d)} \frac{1}{B(u, v)} \\
& \times \int_{0}^{T} \int_{0}^{1} m^{-1+v+L d}(1-m)^{u-1} \exp (-L z \eta m) z^{L d-1} d m d z
\end{aligned}
$$

Using the relationship between the incomplete Gamma function and the general Hyper Geometric function, we obtain

$$
\begin{aligned}
& F_{\mathrm{IB}}(T ; u, v, L, d)=\frac{1}{B(u, v)} \frac{[L T \eta]^{L d}}{\Gamma(L d) L d} \\
& \times \int_{0}^{1} m^{-1+u+L d}(1-m)^{u-1}{ }_{1} F_{1}[L d ; L d+1 ;-L T \eta m] d m
\end{aligned}
$$

That is

$$
\begin{aligned}
& F_{\mathrm{IB}}(T ; u, v, L, d)=\frac{[L T \eta]^{L d+1} \Gamma(L d+1)}{\Gamma^{2}(L d) B(u, v) L d} \\
& \times \int_{0}^{1} m^{v+L d-1}(1-m)^{u-1} G_{1,2}^{1,1}\left(L T m \eta \mid \begin{array}{l}
-L d \\
-1,-L d-1
\end{array}\right) d m
\end{aligned}
$$

Via the integration equation [30],

$$
\begin{aligned}
& \int_{0}^{1} x^{\rho-1}(x-1)^{\sigma-1} G_{p, q}^{m, n}\left(a x \mid \begin{array}{l}
a_{1}, \cdots, a_{p} \\
b_{1}, \cdots, b_{q}
\end{array}\right) d x \\
& =\Gamma(\sigma) G_{p+1, q+1}^{m, n+1}\left(a \begin{array}{ll}
1-\rho, & a_{1}, \cdots, a_{p} \\
b_{1}, \cdots, b_{q}, & 1-\rho-\sigma
\end{array}\right)
\end{aligned}
$$

we obtain

$$
\begin{aligned}
& F_{\mathrm{IB}}(T ; u, v, L, d)=\frac{[L T \eta]^{L d+1} \Gamma(L d+1) \Gamma(v+u)}{\Gamma^{2}(L d) \Gamma(v) L d} \\
& \times G_{2,3}^{1,2}\left(L T \eta \mid \begin{array}{l}
-L d-v,-L d \\
-1,-L d-1,-L d-u-v
\end{array}\right)
\end{aligned}
$$

Therefore, the PDF of the false alarm is

$$
\begin{aligned}
& P_{f a}=1-F_{\mathrm{IB}}(T) \\
& =1-\frac{[L T \eta]^{L d+1} \Gamma(L d+1) \Gamma(v+u)}{\Gamma^{2}(L d) \Gamma(v) L d} \times \\
& G_{2,3}^{1,2}\left(L T \eta \mid \begin{array}{l}
-L d-v,-L d \\
-1,-L d-1,-L d-u-v
\end{array}\right)
\end{aligned}
$$

\section{APPENDIX F}

In B-Wishart case, the unitary $\tilde{\tau}$ obeys the normalized Beta distribution, whose PDF is [23]

$$
\tilde{\tau} \sim \phi_{\mathrm{B}}(u, v)=\frac{1}{B(u, v)} \xi(\xi \tilde{\tau})^{v-1}(1-\xi \tilde{\tau})^{u-1}, \quad \tilde{\tau} \in\left[0, \frac{1}{\xi}\right]
$$

where $\xi=v /(u+\mathrm{v})$, and $u$ and $v$ are the freedom of Beta distribution. $\tau=\xi \tilde{\tau}$ obeys a Beta distribution, whose PDF is

$$
f_{\mathrm{B}}(\tau)=\frac{1}{B(u, v)} \tau^{\nu-1}(1-\tau)^{u-1}
$$

where $u$ and $v$ are the freedom of Beta distribution.

Then, the PDF of the variable $\mathrm{z}$ after an MPWF is

$$
f_{\mathrm{B}}(z ; u, v, L, d)=\int_{0}^{+\infty} \gamma\left(L d, \frac{1}{L}\right) \phi(u, v) J d \tilde{\tau}
$$

where $J=\frac{\partial(x)}{\partial(z)}=\frac{1}{\tilde{\tau}}$. Substitute $\tau=\xi \tilde{\tau}$ and $x=\frac{z}{\tilde{\tau}}$ into Eq.(F.3), and we can obtain

$$
\begin{aligned}
& f_{\mathrm{B}}(z ; u, v, L, d)=\frac{[L z \xi]^{L d}}{z \Gamma(L d)} \frac{1}{B(u, v)} \\
& \times \int_{0}^{1} \tau^{v-L d-1}(1-\tau)^{u-1} \exp \left(-L z \xi \tau^{-1}\right) d \tau
\end{aligned}
$$

Replacing $\tau$ with $1 / m$, we can obtain

$$
\begin{aligned}
& f_{\mathrm{B}}(z ; u, v, L, d)=\frac{[L z \xi]^{L d}}{z \Gamma(L d)} \frac{1}{B(u, v)} \\
& \times \int_{1}^{+\infty} m^{-u-v+L d}(m-1)^{u-1} \exp (-L z \xi m) d m
\end{aligned}
$$

Via the definition of the KummerU function[28], $U(a, b, z) \Gamma(a)=\exp (z) \int_{1}^{+\infty} \exp (-z t)(t-1)^{a-1} t^{b-a-1} d t$,

Eq.(F.5) can be simplified as

$$
\begin{aligned}
& f_{\mathrm{B}}(z ; u, v, L, d)=\frac{[L z \xi]^{L d}}{z \Gamma(L d)} \frac{1}{B(u, v)} \exp (-L z \omega) \\
& \times \Gamma(u) U[u, L d+1-v, L z \xi]
\end{aligned}
$$

Since the KummerU function can be replaced by the WhittakerW function [28], we can obtain

$$
\mathrm{W}_{k, \mu}(x)=x^{\mu+\frac{1}{2}} \exp \left(-\frac{x}{2}\right) U\left(\mu-k+\frac{1}{2}, 2 \mu+1, x\right)(\mathrm{F} .7)
$$

Substituting $k=\frac{L d-v-2 u+1}{2}, \mu=\frac{L d-v}{2}$ and $x=L z \xi$ into Eq.(F.6), then the equation can be expressed in another form

$$
\begin{aligned}
& f_{\mathrm{B}}(z ; u, v, L, d)=z^{\frac{L d+v-3}{2}} \frac{[L \xi]^{\frac{L d+v-1}{2}}}{\Gamma(L d)} \frac{\Gamma(u+v)}{\Gamma(v)} \\
& \times \exp \left(\frac{-L z \xi}{2}\right) \mathrm{W}_{\frac{L d-v-2 u+1}{2}, \frac{L d-v}{2}}(L z \xi)
\end{aligned}
$$

If the threshold is $T$, the CDF should be

$$
\begin{aligned}
& F_{\mathrm{B}}(T ; u, v, L, d)=\frac{[L \xi]^{L d}}{\Gamma(L d)} \frac{1}{B(u, v)} \\
& \times \int_{0}^{T} \int_{1}^{+\infty} m^{-u-v+L d}(m-1)^{u-1} \exp (-L z \xi m) z^{L d-1} d m d z
\end{aligned}
$$

where $L$ is the number of looks, $d$ is the dimension of the scattering vector, $\omega$ is the mean of the texture $\tau, u$ and $v$ are the freedom parameters of the Beta distribution, and $m=1 / \tau$. The relationship between the incomplete Gamma function and the hypergeometric function [28] is

$$
\Gamma(a, x)=\int_{0}^{x} e^{-t} t^{a-1} d t=a^{-1} x^{a}{ }_{1} F_{1}(a ; a+1 ;-x)
$$

Instituting Eq. (F.10) into Eq. (F.9), we obtain 


$$
\begin{aligned}
& F_{\mathrm{B}}(T ; u, v, L, d)=\frac{1}{B(u, v)} \frac{[L T \xi]^{L d}}{\Gamma(L d) L d} \\
& \times \int_{1}^{+\infty} m^{-v-u+L d}(m-1)^{u-1}{ }_{1} F_{1}[L d ; L d+1 ;-L T \xi m] d m
\end{aligned}
$$

The relationship between the general hypergeometric function and the MeijerG-function is

$$
\begin{aligned}
& { }_{p} F_{q}\left(a_{1}, \cdots, a_{p} ; b_{1}, \cdots, b_{q} ;-x\right)=\frac{\prod_{j=1}^{q} \Gamma\left(b_{j}\right)}{\prod_{j=1}^{p} \Gamma\left(a_{j}\right)} \\
& \times x G_{p, q+1}^{1, p}\left(x \mid \begin{array}{l}
-a_{1}, \cdots,-a_{p} \\
-1,-b_{1}, \cdots,-b_{q}
\end{array}\right) \quad p \leq q+1
\end{aligned}
$$

where $G_{p, q}^{m, n}\left(x \mid \begin{array}{l}a_{1}, \cdots, a_{p} \\ b_{1}, \cdots, b_{q}\end{array}\right)$ is the MeijerG-function. -Instituting

Eq. (F.12) into Eq. (F.11), we obtain

$$
\begin{aligned}
& F_{\mathrm{B}}(T ; u, v, L, d)=\frac{[L T \xi]^{L d+1} \Gamma(L d+1)}{\Gamma^{2}(L d) B(u, v) L d} \\
& \times \int_{1}^{+\infty} m^{-v-u+L d+1}(m-1)^{u-1} G_{1,2}^{1,1}\left(L T m \xi \mid \begin{array}{l}
-L d \\
-1,-L d-1
\end{array}\right) d m
\end{aligned}
$$

If we have the integration equation[30]:

$$
\begin{aligned}
& \int_{1}^{\infty} x^{-\rho}(x-1)^{\sigma-1} G_{p, q}^{m, n}\left(a x\left(\begin{array}{l}
a_{1}, \cdots, a_{p} \\
b_{1}, \cdots, b_{q}
\end{array}\right) d x\right. \\
& =\Gamma(\sigma) G_{p+1, q+1}^{m+1, n}\left(a \begin{array}{l}
a_{1}, \cdots, a_{p}, \rho \\
\rho-\sigma, b_{1}, \cdots, b_{q}
\end{array}\right)
\end{aligned}
$$

Eq. (F.13) can be converted into

$$
\begin{aligned}
& F_{\mathrm{B}}(T ; u, v, L, d)=\frac{[L T \xi]^{L d+1} \Gamma(L d+1) \Gamma(v+u)}{\Gamma^{2}(L d) \Gamma(v) L d} \\
& \times G_{2,3}^{2,1}\left(L T \xi \mid \begin{array}{l}
-L d, v+u-L d-1 \\
v-L d-1,-1,-L d-1
\end{array}\right)
\end{aligned}
$$

and the false alarm probability can be converted into

$$
\begin{aligned}
& P_{f a}=1-F_{\mathrm{B}}(T ; u, v, L, d) \\
& =1-\frac{[L T \xi]^{L d+1} \Gamma(L d+1) \Gamma(v+u)}{\Gamma^{2}(L d) \Gamma(v) L d} \\
& \times G_{2,3}^{2,1}\left(L T \xi \mid \begin{array}{l}
-L d, v+u-L d-1 \\
v-L d-1,-1,-L d-1
\end{array}\right)
\end{aligned}
$$

\section{APPENDIX G}

The unitary textural variable $\tilde{\tau}$ obeys the normalized Beta distribution, whose PDF is [24]

$$
\tilde{\tau} \sim f(\tilde{\tau} ; \kappa, \nu)=\frac{|\nu| \kappa^{\kappa}}{\sigma \Gamma(\kappa)}\left(\frac{\tilde{\tau}}{\sigma}\right)^{\kappa \nu-1} \exp \left\{-\kappa\left(\frac{\tilde{\tau}}{\sigma}\right)^{\nu}\right\}
$$

where $\sigma=\kappa^{1 / \nu} \Gamma(\kappa) / \Gamma(\kappa+1 / \nu)$, and $\sigma, \kappa, \nu$ are the scale, shape and power parameters of GГD distribution. Then, the $\mathrm{PDF}$ of the variable $\mathrm{z}$ after an MPWF is

$$
\begin{aligned}
& f_{\mathrm{H}}(z ; \kappa, \nu, L, d)=\int_{0}^{+\infty} f(z, \tilde{\tau}) d \tilde{\tau}=\int_{0}^{+\infty} f(x, \tilde{\tau}) J d \tilde{\tau} \\
& =\int_{0}^{+\infty} \gamma\left(L d, \frac{1}{L}\right) f(\tilde{\tau} ; \kappa, \nu) J d \tilde{\tau}
\end{aligned}
$$

where $J=\frac{\partial(x)}{\partial(z)}=\frac{1}{\tilde{\tau}}$. And we have the equation

$$
\begin{aligned}
& Z_{\rho}^{v}=\int_{o}^{\infty} t^{\nu-1} \exp \left[-t^{\rho}-\frac{z}{t}\right] d t \\
& =\left\{\begin{array}{cl}
\frac{1}{\rho} H_{0,2}^{2,0}[z \mid \\
\left.(0,1),\left(\frac{v}{\rho}, \frac{1}{\rho}\right)\right] & \rho>0 \\
-\frac{1}{\rho} H_{1,1}^{1,1}\left[z\left[\begin{array}{c}
\left(1-\frac{v}{\rho},-\frac{1}{\rho}\right) \\
(0,1)
\end{array}\right]\right. & \rho<0
\end{array}\right.
\end{aligned}
$$

Substituting $x=\frac{z}{\tilde{\tau}}$ into Eq.(G.2), and using the following relation [31]

$$
z^{\sigma} H_{p, q}^{m, n}\left[z \mid \begin{array}{l}
\left(a_{p}, A_{p}\right) \\
\left(b_{q}, B_{q}\right)
\end{array}\right]=H_{p, q}^{m, n}\left[z \mid \begin{array}{l}
\left(a_{p}+\sigma A_{p}, A_{p}\right) \\
\left(b_{q}+\sigma B_{p}, B_{q}\right)
\end{array}\right]
$$

we can obtain

$$
\begin{aligned}
& f_{\mathrm{H}}(z ; \kappa, \nu, L, d)==\int_{0}^{+\infty} \gamma(d, L d) f(\tilde{\tau} ; \kappa, \nu) J d \tilde{\tau} \\
& =\frac{|v| \kappa^{\kappa} L^{L d} z^{L d-1}}{\Gamma(L d) \Gamma(\kappa) \sigma^{k v}} \int_{0}^{+\infty} \tilde{\tau}^{\kappa v-L d-1} \exp \left\{-L z \tilde{\tau}^{-1}-\frac{\kappa \tilde{\tau}^{v}}{\sigma^{v}}\right\} d \tilde{\tau} \\
& =\left\{\begin{array}{c}
\frac{\kappa^{1 / v} L}{\Gamma(L d) \Gamma(\kappa) \sigma} H_{0,2}^{2,0}\left[\frac{L z \kappa^{1 / v}}{\sigma} \mid \begin{array}{c}
(L d-1,1) \quad\left(\kappa-\frac{1}{v}, \frac{1}{v}\right)
\end{array}\right] v>0 \\
\frac{\kappa^{1 / v} L}{\Gamma(L d) \Gamma(k) \sigma} H_{1,1}^{1,1}\left[\frac{L z \kappa^{1 / v}}{\sigma} \mid \begin{array}{c}
\left(1-\kappa+\frac{1}{v},-\frac{1}{v}\right) \\
(L d-1,1)
\end{array}\right] \quad v<0
\end{array}\right.
\end{aligned}
$$

Via the following equation [31]

$$
\begin{aligned}
& \int_{0}^{t} x^{\rho-1}(t-x)^{\sigma-1} H_{p, q}^{m, n}\left[b x^{k} \mid \begin{array}{c}
\left(a_{p}, A_{p}\right) \\
\left(b_{q}, B_{q}\right)
\end{array}\right] d x \\
& =t^{\rho+\sigma-1} \Gamma(\sigma) H_{p+1, q+1}^{m, n+1}\left[b t^{k} \mid \begin{array}{c}
(1-\rho, k),\left(a_{p}, A_{p}\right) \\
\left(b_{q}, B_{q}\right),(1-\rho-\sigma, k)
\end{array}\right]
\end{aligned}
$$

we can get the cdf

$$
F_{\mathrm{H}}(T ; \kappa, \nu, L, d)=\int_{0}^{T} f_{\mathrm{H}}(z ; \kappa, \nu, L, d) d z=\frac{T \kappa^{1 / v} L}{\Gamma(L d) \Gamma(\kappa) \sigma}
$$

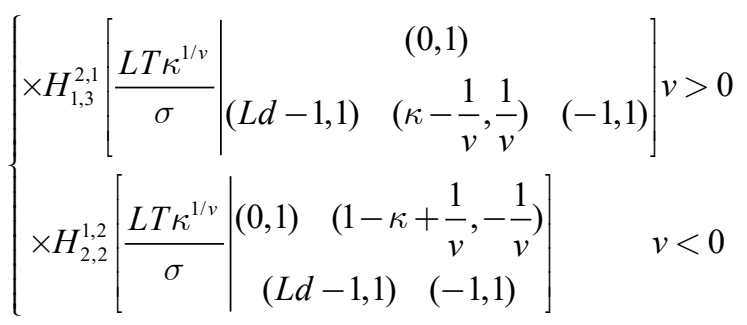


and the false alarm probability can be converted into

$$
P_{f a}=1-F_{\mathrm{H}}(T ; \kappa, \nu, L, d)
$$

where $P_{f a}$ is the false alarm rate, $T$ is the detection threshold, $L$ is the equivalent number of looks, $d$ is the dimension of the polarimetric scattering vector, $\Gamma(\cdot)$ is the Gamma function, $H(\cdot)$ is the Fox $\mathrm{H}$ function and $\sigma, \kappa, \nu$ are the scale, shape and power parameters of GГD distribution.

\section{REFERENCE}

[1] A. Marino, S. R. Cloude, and I. H. Woodhouse, "Detecting depolarized targets using a new geometrical perturbation filter," IEEE Transactions on Geoscience and Remote Sensing., vol. 50, no. 10, pp. 3787-3799, Oct. 2012.

[2] L. M. Novak, M. B. Sechtin and M. J. Cardullo, "Studies of target detection algorithms that use polarimetric radar data," IEEE Transactions on Aerospace and Electronic Systems, vol. 25, no. 2, pp. 150-165, March 1989.

[3] L. M. Novak and M. C. Burl, "Optimal speckle reduction in polarimetric SAR imagery," IEEE Transactions on Aerospace and Electronic Systems., vol. 26, no. 2, pp. 293-305, Mar. 1990.

[4] A. Marino, "A notch filter for ship detection with polarimetric SAR data," IEEE Journal of Selected Topics in Applied Earth Observation and Remote Sensing, vol. 6, no. 10, pp. 1219-1232, 2013.

[5] A. Marino, Sugimoto, Mitsunobu, and Ouchi, et al., "Validating a notch filter for detection of targets at sea with ALOS-PALSAR data: Tokyo Bay," IEEE Journal of Selected Topics in Applied Earth Observation and Remote Sensing, vol. 7, no. 12, pp. 4907-4918, Dec. 2014.

[6] A. Marino and I. Hajnsek, "Statistical tests for a ship detector based on the polarimetric notch filter," IEEE Transactions on Geoscience and Remote Sensing., vol. 53, no. 8, pp. 4578-4595, Aug. 2015.

[7] G. Gao and G. Shi, "CFAR Ship Detection in Nonhomogeneous Sea Clutter Using Polarimetric SAR Data Based on the Notch Filter," IEEE Transactions on Geoscience and Remote Sensing., vol. 55, no. 8, pp. 4811-4824, Aug. 2017.

[8] S. R. Cloude and E. Pottier, "A review of target decomposition theorems in radar polarimetry," IEEE Trans. Geosci. Remote Sens., vol. 34, no. 2, pp. 498-518, Mar. 1996.

[9] R. Ringrose and N. Harris, "Ship detection using polarimetric SAR data," in Proc. Eur. Space Agency (ESA-SP), vol. 450, Mar. 2000, pp. 687.

[10] J. Chen, Y. Chen, and J. Yang, "Ship detection using polarization crossentropy," IEEE Geosci. Remote Sens. Lett., vol. 6, no. 4, pp. 723-727, Oct. 2009.

[11] Z. Xu, B. Tang and S. Cheng, "Faint Ship Wake Detection in PolSAR Images," IEEE Geoscience and Remote Sensing Letters, vol. 15, no. 7, pp. 1055-1059, July 2018.

[12] F. Biondi, "Low-Rank Plus Sparse Decomposition and Localized Radon Transform for Ship-Wake Detection in Synthetic Aperture Radar Images," IEEE Geoscience and Remote Sensing Letters., vol. 15, no. 1, pp. 117-121, Jan. 2018.

[13] F. Biondi, "A Polarimetric Extension of Low-Rank Plus Sparse Decomposition and Radon Transform for Ship Wake Detection in Synthetic Aperture Radar Images," IEEE Geoscience and Remote Sensing Letters., vol. 16, no. 1, pp. 75-79, Jan. 2019.

[14] A. De Maio, D. Orlando, and L. Pallotta, et al., "A Multifamily GLRT for Oil Spill Detection," IEEE Transactions on Geoscience and Remote Sensing., vol. 55, no. 1, pp. 63-79, Jan. 2017.

[15] L. M. Novak, M. C. Burl, and W.W. Irving. et al, "Optimal polarimetric processing for enhanced target detection," in Proc. Telesystems Conference Proceedings, Oct. 1991, pp. 69-75.

[16] A. Lopes and F. Sery, "Optimal speckle reduction for the product model in multilook polarimetric SAR imagery and the Wishart distribution," IEEE Transactions on Geoscience and Remote Sensing., vol. 35, no. 3, pp. 632-647, May.1997.

[17] G. Liu, S. Huang, and A. Torre, et al., "The multilook polarimetric whitening filter (MPWF) for intensity speckle reduction in polarimetric SAR images," IEEE transactions on geoscience and remote sensing., vol. 36, no. 3, pp. 1016-1020, May. 1998.

[18] G. Liu, X. Hong, and S. Huang, "An extension of multi-look polarimetric whitening filters for speckle reduction in polarimetric SAR imagery," in Proc. IEEE International Geoscience and Remote Sensing Symposium, Jul. 1998, pp. 27-29.
[19] S. N. Anfinsen, T. Ding, and C. Brekke, "Improved target detection in polarimetric SAR images by use of mellin kind statistics," in Proc. International Workshop on Science and Applications of SAR Polarimetry and Polarimetric Interferometry, Jan. 2011.

[20] W. Gao, F. Yang, and Y. Cui, "The extended polarimetric whitening filter and its application to target detection in polarimetric synthetic aperture radar images," IEEE Geoscience and Remote Sensing Letters., vol. 13, no. 3, pp. 419-423, 2016.

[21] D. Tao, S. N. Anfinsen and C. Brekke, "Robust CFAR Detector Based on Truncated Statistics in Multiple-Target Situations," IEEE Transactions on Geoscience and Remote Sensing., vol. 54, no. 1, pp. 117-134, Jan. 2016.

[22] G. Liu, S. Huang, and A. Torre, et al, "Optimal speckle reduction in multi-look polarimetric SAR image," in Proc. Geoscience and Remote Sensing Symposium, 1995, pp. 664-666.

[23] L. Bombrun, S.N. Anfinsen, and O. A. Harant, "Complete coverage of log-cumulant space in terms of distributions for Polarimetric SAR data," In Proc. 5th International Workshop on Science and Applications of SAR Polarimetry and Polarimetric Interferometry(POLinSAR2011), Jan. 2011, pp. 1-8.

[24] H. Li, W. Hong, Y. Wu and P. Fan, "An Efficient and Flexible Statistical Model Based on Generalized Gamma Distribution for Amplitude SAR Images," IEEE Transactions on Geoscience and Remote Sensing., vol. 48, no. 6, pp. 2711-2722, June. 2010

[25] S. N. Anfinsen, A. P. Doulgeris and T. Eltoft, "Estimation of the Equivalent Number of Looks in Polarimetric Synthetic Aperture Radar Imagery," IEEE Transactions on Geoscience and Remote Sensing., vol. 47, no. 11, pp. 3795-3809, Nov. 2009.

[26] Lee. J. S., Schuler, D. L., Lang, R. H. and Ranson, K. J., "K distribution for multilook processed polarimetric SAR imagery," Geoscience and Remote Sensing Symposium, 1994. IGARSS '94. 'Surface and Atmospheric Remote Sensing: Technologies, Data Analysis and Interpretation'., International, vol. 4, Aug. 1994, pp. 2179-2181.

[27] S. Khan, and R. Guida, "On Fractional Moments of Multilook Polarimetric Whitening Filter for Polarimetric SAR Data," IEEE Transactions on Geoscience and Remote Sensing., vol. 52, no. 6, pp. 3502-3512, 2014.

[28] M. Abramowitz, I. A. Stegun, Handbook of Mathematical Functions With Formulas, Graphs, and Mathematical Tables, Dover Publications Inc., NYC, USA, 2001

[29] D. Nestor, "On the distribution of the sum of gamma-gamma variates and applications in RF and optical wireless communications," IEEE Transactions on Communications., vol. 59, no. 5, pp. 1298-1308, 2011.

[30] I. S. Gradshteyn, I. M. Ryzhik, Table of integrals, series, and products, Academic Press, San Diego, CA 2007.

[31] A. Mathai, R Saxena, H. Haubold, The H-function: Theory and Applications, Springer, NYC, USA, 2010.

[32] L. Tao, C. Haogui, X. Ze-min, et al., "On Log-Cumulants of Multilook Polarimetric Whitening Filter for Polarimetric SAR Data," IET Radar, Sonar \& Navigation., vol. 10, no. 4, pp. 655-662, 2016.

[33] S. N. Anfinsen, and T. Eltof, "Application of the Matrix-variate Mellin transform to analysis of polarimetric radar images," IEEE Transactions on geoscience and remote sensing., vol. 49, no .6, pp. 2281-2295, 2011.

[34] S. N. Anfinsen, A. P. Doulgeris and T. Eltoft, "Goodness-of-Fit Tests for Multilook Polarimetric Radar Data Based on the Mellin Transform," IEEE Transactions on Geoscience and Remote Sensing, vol. 49, no. 7, pp. 2764-2781, July 2011.

[35] V. A. Krylov et al., "On the Method of Logarithmic Cumulants for Parametric Probability Density Function Estimation," IEEE Transactions on Image Processing, vol. 22, no. 10, pp. 3791-3806, Oct. 2013.

[36] J. Wei, P. Li, and J. Yang, et al., "A new automatic ship detection method using L-band polarimetric SAR imagery," IEEE Journal of Selected Topics in Applied Earth Observations and Remote Sensing., vol. 7, no. 4, pp. 1383-1393, May, 2017.

[37] J. Martín-de-Nicolás et al., "Robustness of a Generalized Gamma CFAR ship detector applied to TerraSAR-X and Sentinel-1 images," in Conf. IEEE EUROCON 2015 - International Conference on Computer as a Tool (EUROCON), Salamanca, 2015, pp. 1-6.

[38] L. Tao, C. Haogui, X. Ze-min, and G. Jun, "Novel Estimators of Equivalent Number of looks in Polarimetric SAR imagery based on Sub-matrices," Science China Information Science., vol. 59, no. 6, pp. 1869-1919, 2016

[39] M. Ester, "A Density-based Algorithm for Discover erring Clusters in Large Spatial Databases with Noise," In Proc. $2^{\text {nd }}$ International Conference on Know ledge Discovery and Data Mining. Aug. 1996.

[40] R. L. Paes, J. A. Lorenzzetti, and D. F. M. Gherardi, "Ship detection 
using TerraSAR-X images in the campo basin (Brazil)," IEEE Geosci. Remote Sens. Lett., vol. 7, no. 3, pp. 545-548, Jul. 2010.

[41] T.A. Tsiftsis, "Performance of heterodyne wireless optical communication systems over gamma-gamma atmospheric turbulence channels," Electronics Letters., vol. 44, no. 5, pp. 372-373. Mar, 2008.

[42] H. Bateman, Tables of integral transforms. McGraw-Hill, NYC, USA, 1954.

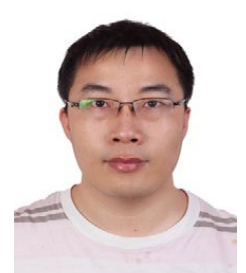

Tao Liu received the B.S. degree in communication engineering and the Ph.D. degree in information and communication engineering, all from the National University of Defense Technology (NUDT), Changsha, China, in 2001 and 2007 respectively.

In 2000, he was the Outstanding Communist Youth League member of China. Since 2007, he has been with the School of Electronic Engineering, Naval University of Engineering, where he is currently a Professor. In 2009 he received the Award of Excellent Doctor Thesis of Chinese Army. Since 2019 he is a committee member in Radar Branch of the Chinese Institute of Electronics (CIE).

He has authored over 50 journal papers and three books. His research interests include statistical theory of radar polarization, polarization information processing, synthetic aperture radar (SAR) automatic target recognition, statistical modeling of SAR image, SAR ship detection, InSAR (interferometric SAR), SAR ground moving target indication and Artificial Intelligence(AI).

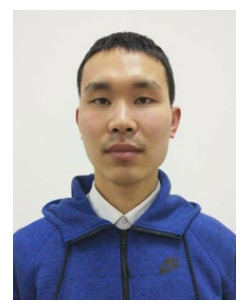

Jiafeng Yang received the B.S. degree in radar engineering and the M.S. degree in signal processing, all from the Naval University of Engineering (NUE), Wuhan, China, in 2015 and 2018 respectively.

His research interests include radar polarization information process and electronic warfare system modeling and SAR ground moving target indication.

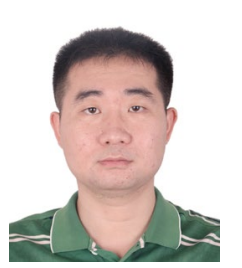

Gui Gao (M'09) received the B.S. in information engineering, the M.S. and Ph.D. degrees in remote sensing information processing from National University of Defense Technology (NUDT), Changsha, China, in 2002, 2003 and 2007, respectively. From 2007, he joined the Faculty of Information Engineering, School of Electronic Science and Engineering, NUDT, as an associate professor. From 2017, he was with Faculty of Geosciences and Environmental Engineering, Southwest Jiaotong University, Chengdu, China, where he is currently a professor. From 2018, he was also with the College of Traffic Engineering, Hunan University of Technology, Zhuzhou, China, where he is currently a distinguished professor. He has authored over 100 journal and conference papers and has written four books and an English chapter. His current research interests include radar signal processing, InSAR (interferometric SAR), target detection, marine environment, and SAR GMTI (ground moving target indication).
Dr. Gao is a member of the IEEE Geoscience and Remote Sensing Society, the Applied Computational Electromagnetics Society, and a senior member of the Chinese Institute of Electronics (CIE), and a Dominant Member of Young Scientist Forum of CIE. He received the Award of Excellent Master Thesis of Hunan Province in 2006, the Award of Excellent Doctor Thesis of Chinese Army in 2008, Awards of Outstanding Young People in NUDT and Hunan Province of China in 2014 and 2016, a first Class Prize of Chinese Military Science and Technology Progress Award, and the Award of Natural Science in Hunan Province. He was also supported by the Excellent Young People Science Foundation of the National Natural Science Foundation of China. $\mathrm{He}$ is the Leader Guest Editor of the International Journal of Antenna and Propagation, the Associate Editor and Leader Guest Editor of the IEEE Journal of Selected Topics in Applied Earth Observations and Remote Sensing.

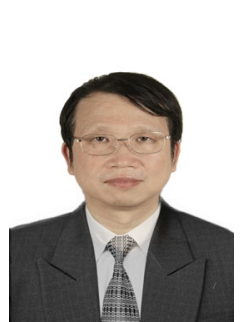

Jian Yang (M'03, SM' 04) received the B.S. and M.S. degrees from Northwestern Polytechnical University, Xian, China, in 1985 and 1990, respectively, and the Ph.D. degree from Niigata University, Niigata, Japan, in 1999. In 1985, he joined the Department of Applied Mathematics, Northwestern Polytechnical University. From 1999 to 2000, he was an Assistant Professor with Niigata University. In April 2000, he joined the Department of Electronic Engineering, Tsinghua University, Beijing, China, and he was promoted to a full Professor in 2002. He has published more than 300 papers and received many awards. His research interesting areas include radar polarimetry, feature extraction, target detection and target classification.

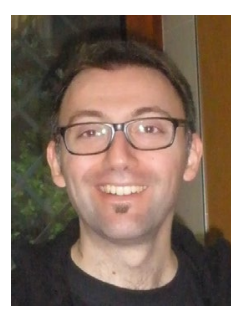

Armando Marino (M'2011) received the M.Sc. degree in telecommunication engineering from the Universita' di Napoli "Federico II," Naples, Italy, in 2006. In 2006, he joined the High Frequency and Radar Systems Department, German Aerospace Centre, Oberpfaffenhofen, Germany, where he developed his M.Sc. thesis. He received the Ph.D. degree in polarimetric SAR interferometry from the School of Geosciences, University of Edinburgh, Edinburgh, U.K., in 2011. From March 2011 to October 2011, he was with the University of Alicante, Institute of Computing Research, Spain. From December 2011 to May 2015, he was a Postdoctoral Researcher and Lecturer with ETH Zurich, Institute of Environmental Engineering, Switzerland. From June 2015, he was a Lecturer with the School of Engineering and Innovation, Open University, Milton Keynes, U.K. Since May 2018 he is a Senior Lecturer (Assistant Professor) at the University of Stirling, Faculty of Natural Sciences, Stirling, UK.

Dr. Marino's Ph.D. thesis received the "Best Ph.D. Thesis 2011 " by the Remote Sensing and Photogrammetry Society and the "Outstanding Ph.D. Thesis" by Springer Verlag, which published the thesis in 2012. 